\title{
Potential vorticity: Measuring consistency between GCM dynamical cores and tracer advection schemes
}

\author{
J. P. Whitehead, ${ }^{\mathrm{a}, \mathrm{b} *}$ C. Jablonowski, ${ }^{\mathrm{b}}$ J. Kent $^{\mathrm{b}}$ and R. B. Rood ${ }^{\mathrm{b}}$ \\ ${ }^{a}$ Mathematics Department, Brigham Young University, Provo, UT, USA \\ ${ }^{\mathrm{b}}$ Department of Atmospheric, Oceanic and Space Sciences, University of Michigan, Ann Arbor, MI, USA
}

${ }^{\star}$ Correspondence to: J. P. Whitehead, Mathematics Department, Brigham Young University, 275 TMCB, Provo, UT 84602, USA.

E-mail: whitehead@math.byu.edu

\begin{abstract}
Ertel's potential vorticity (PV) is used as a diagnostic tool to give a direct comparison between the treatment of $\mathrm{PV}$ in the dynamics and the integration of $\mathrm{PV}$ as a passive tracer, yielding a systematic evaluation of a model's consistency between the dynamical core's integration of the equations of motion and its tracer transport algorithm. Several quantitative and qualitative metrics are considered to measure the consistency, including error norms and grid-independent probability density functions. Comparisons between the four dynamical cores of the National Center for Atmospheric Research's (NCAR) Community Atmosphere Model version 5.1 (CAM) are presented. We investigate the consistency of these dynamical cores in an idealized setting: the presence of a breaking baroclinic wave. For linear flow, before the wave breaks, the consistency for each model is good. As the flow becomes nonlinear, the consistency between dynamic PV and tracer PV breaks down, especially at small scales. Large values of dynamic PV are observed that do not appear in the tracer PV. The results indicate that the spectral-element (CAM-SE) dynamical core is the most consistent of the dynamical cores in CAM, however the consistency between dynamic PV and tracer PV is related to and sensitive to the diffusive properties of the dynamical cores.
\end{abstract}

Key Words: dynamical core; tracer transport; potential vorticity; advection; baroclinic wave

Received 2 July 2012; Revised 11 March 2013; Accepted 24 April 2014; Published online in Wiley Online Library 17 June 2014

\section{Introduction}

Much attention has been paid of late to the evaluation and accuracy of the dynamical cores of general circulation models (GCMs). The term dynamical core refers to the integration of the nonlinear equations of motion and typically includes all transport processes. One of the key building blocks for a dynamical core is the advection scheme which passively advects the many (possibly hundreds) of tracers used in climate studies (Lamarque et al., 2008). Tracer advection schemes implicitly rely on the accurate integration of the momentum equation because the advective winds are taken from this dynamic step. Some models even use the identical tracer advection algorithm as a building block for integrating both the tracers and the momentum equations (Lin and Rood, 1996; Lin, 2004). Tracer advection routines and the integration of the nonlinear dynamics are therefore fundamentally linked. The impact that this relationship has on the subgrid (unresolved) scales needs further quantification. The current article is one approach to this problem.

Investigations into the veracity of a model's tracer transport algorithm (Lauritzen et al., 2011) are necessary to validate model performance. Typically, such test cases are performed on a variety of modelling frameworks, isolating the effect of the advective transport via a series of tests with variable difficulty
(Nair and Lauritzen, 2010; Kent et al., 2012a, 2014). These tests have prescribed dynamical fields, such as prescribed wind velocities, and omit the parametrized physics, concentrating on the advection algorithm. Other tests such as the adiabatic baroclinic wave test by Jablonowski and Williamson (2006a) provide a means to compare the evolution of the nonlinear dynamics (as opposed to the linear tracer advection equation) between models, while omitting the effect of complicated physics parametrizations. In a different approach to examining the dynamical core or tracer advection routine separately, Rasch et al. (2006) closely monitored the effect of different tracer routines on climate-related constituents, using the full physics parametrization package available in the National Center for Atmospheric Research (NCAR)'s Community Atmosphere Model (CAM) version 3.0. Rasch et al. (2006) considered three of the dynamical cores available in CAM. These are named after the discretization method of the prognostic equations in the dynamical core: finite volume (CAM-FV), spectral-transform Eulerian (CAM-EUL), and spectral-transform semi-Lagrangian (CAM-SLD). They found that, even with the identical physics parametrization package, the evolution of the tracers depended on the choice of dynamical core and hence the choice of the advection algorithm. By investigating the relationship between the discrete integration of the dynamics and the corresponding 
tracer transport algorithm within each of the four dynamical cores in CAM version 5.1 (Neale et al., 2010), we shed some light on the results of Rasch et al. (2006).

In Williamson (2007) it is noted that employing two different numerical schemes for the integration of the dynamics and for tracer advection 'is not entirely satisfactory'. This aspect is, for example, illustrated in Zhang et al. (2008). They observed that a tracer advection scheme that was not inherently consistent with the dynamical framework in the atmospheric model GAMIL (Wang et al., 2004; Wan et al., 2006) led to significant errors in a radon transport test. Adjusting the advective scheme appropriately appeared to reduce the impact of these errors, and Zhang et al. (2008) proposed that the difference arose from the lack of consistency of the originally used advective scheme. These results highlight only some of the possible issues that may arise from treating advection differently than the dynamics (Lee et al., 2004; Lauritzen et al., 2011). Furthermore, Joeckel et al. (2001) emphasized these ideas, arguing that, for accurate constituent transport, the discrete advective continuity equation should reduce to that used for the transport of mass (dynamics). This restriction on the design of a dynamical core was one of the primary considerations in the design of CAM-FV (Lin and Rood, 1996; Lin, 2004; Rood, 2011). In this article we suggest an idealized approach which measures this 'consistency' between the dynamical core and its tracer advection scheme. This is done via the assessment of Ertel's potential vorticity which acts both as a dynamic quantity and passive tracer.

Ertel's potential vorticity (PV) with symbol $q$ is defined as

$$
q=\frac{1}{\rho}(2 \boldsymbol{\Omega}+\nabla \times \boldsymbol{u}) \cdot(\nabla \theta)
$$

where $\rho$ is the density of the air, $\mathbf{u}$ stands for the three-dimensional velocity vector field, $\boldsymbol{\Omega}$ symbolizes the Earth's rotation vector, $\theta$ denotes the potential temperature, and $\nabla \times$ and $\nabla$ are the curl and gradient operators. When the hydrostatic and shallowatmosphere approximations are made, as is the case for the hydrostatic primitive equations, then the isobaric and isentropic versions of Eq. (1) are

$$
\begin{aligned}
& q=-g\left(f \widehat{\mathbf{k}}+\nabla_{p} \times \boldsymbol{v}\right) \cdot \nabla_{p} \theta \\
& q=-g\left(f+\widehat{\mathbf{k}} \cdot \nabla_{\theta} \times \boldsymbol{v}\right) \frac{\partial \theta}{\partial p}
\end{aligned}
$$

respectively (Hoskins et al., 1985). Here, $g$ symbolizes the gravity, $\widehat{\mathbf{k}}$ is the vertical unit vector, $f=2 \Omega \sin \phi$ is the Coriolis parameter with the scalar angular velocity of the Earth $\Omega, \phi$ represents latitude, $\mathbf{v}$ is the horizontal velocity field $\mathbf{v}=(u, v, 0)$ with the zonal and meridional wind components $u$ and $v$, $\nabla_{p} \times$ and $\nabla_{p}$ are the three-dimensional curl and gradient operators applied on levels of constant pressure $p$, and $\nabla_{\theta}$ is the three-dimensional gradient operator applied along levels of constant potential temperature. For adiabatic, frictionless flow, the potential vorticity is conserved following the flow (Ertel, 1942; Hoskins et al., 1985; Salmon, 1998; Gibbon and Holm, 2010) which yields a tracer advection equation for $q$

$$
\begin{aligned}
\frac{\mathrm{D} q}{\mathrm{D} t} & =0, \\
\Leftrightarrow \frac{\partial q}{\partial t}+\boldsymbol{u} \cdot \nabla q & =0,
\end{aligned}
$$

with $\mathrm{D} / \mathrm{D} t$ representing the material derivative and $t$ time. For completeness, we note that the flux form of this conservation is given by

$$
\frac{\partial(\rho q)}{\partial t}+\nabla \cdot(\boldsymbol{u} \rho q)=0
$$

As an aside, even diabatic effects like friction (as a general term for all dissipative processes) can be formally included into the PV equations (4) and (6) as e.g. detailed in Haynes and McIntyre (1987, 1990). It yields

$$
\begin{aligned}
\frac{\mathrm{D} q}{\mathrm{D} t} & =-\frac{1}{\rho} \nabla \cdot \mathbf{N} \\
\frac{\partial \rho q}{\partial t}+\nabla \cdot(\boldsymbol{u} \rho q+\mathbf{N}) & =0
\end{aligned}
$$

in either Lagrangian or flux form, respectively, where $\mathbf{N}$ denotes a non-advective flux that is driven by e.g. subgrid-scale diffusive processes. The exact form of $\mathbf{N}$ and how frictional forces enter this vector term is thoroughly discussed in Haynes and McIntyre (1990), but is not relevant here. The important point is that Eqs (7) and (8) do not contain any source or sink terms in the classical, e.g. chemical, sense. Rather, the diabatic term on the right-hand side of Eq. (7) appears as the specific volume times a divergence and thereby denotes a non-advective redistribution (transport) process as clearly shown by the flux form in Eq. (8). Such a redistribution is conservative. Therefore, our measure of consistency is also applicable to dynamical cores and tracer advection algorithms in the presence of subgrid-scale dissipation $\mathbf{N}$, whether explicitly added or implicitly induced via the numerical scheme. We emphasize that the type of dissipation in the discretized tracer advection algorithm and the dynamical core is most often different. This indicates that the subgrid-scale dissipation may cause inconsistencies between the dynamic PV and its passive tracer PV counterpart, which is at the very essence of this article.

Note that there is a substantial body of work on understanding atmospheric dynamics, tracer transport and residence times of chemically and radiatively important trace gases which relies on the correlative relationship of potential vorticity and several tracers (e.g. Newman et al., 1988). One goal of this work is to explore the ability of models to represent the relationship between potential vorticity and tracers, and hence evaluate the model's ability to represent these important environmental issues and develop strategies to improve the models in this regard. In addition, it has been well noted that the reversal of the meridional PV gradient serves as a necessary condition for baroclinic instability as discussed by Charney and Stern (1962), and Hoskins et al. (1985) emphasized the invertibility principle of the PV distribution. This further demonstrates the importance of accurately predicting the PV distribution, even beyond the tracer-dynamics consistency arguments in this article.

From the equations above, the key observation for this article is that PV is conserved along isentropes that do not intersect the ground. Hence, given initial data denoted by $\mathbf{u}_{0}, \theta_{0}, \rho_{0}$, we can define the initial PV field $q_{0}$ using Eq. (1) which can be advected via Eq. (4), (5) or (6). The same applies to the hydrostatic PV representations (Eqs (2) and (3)) provided that $\mathbf{v}_{0}$ and $\theta_{0}$ are initially known at either pressure or isentropic levels. At any given time $t$, the solution $q(t)$ should be identical to the potential vorticity computed from the dynamical variables $\mathbf{u}(t)$, $\theta(t)$ and $\rho(t)$ which are solutions of the dynamical equations of motion (the hydrostatic primitive equations if Eq. (2) or (3) are used) with initial conditions $\mathbf{u}_{0}, \theta_{0}, \rho_{0}$. Therefore, a model that purports to maintain consistency between tracer advection and the integration of the dynamical equations should ensure that a tracer initialized as PV is identical to PV computed from the dynamic variables. Using PV both as a tracer and a diagnostic computed from the prognostic variables then allows a direct evaluation of a given model's ability to maintain accurate relationships between tracers and dynamic variables.

Such considerations are not entirely new, as Davis et al. (1993) compared the evolution of a PV tracer to the diagnostic PV to evaluate the relative effects of latent heating and friction on the evolution of a cyclone. Davis et al. (1993) used the invertibility principle (Hoskins et al., 1985) for appropriately balanced flows to then deduce the influence of diabatic processes on the wind and temperature fields. This idea is carried further in Brennan 
et al. (2007) where the non-conservation of PV is utilized to diagnose the influence of latent heat release on the development of low-level jets and cyclones. The use of a PV tracer is further explored in Stoelinga (1996), Gray (2006), Chagnon and Gray (2009) and Chagnon et al. (2013). A detailed explanation of the methodology pursued in these studies is given in Chagnon et al. (2013). The essential concept is to introduce a series of tracers into the model evolution that have as sources the calculated diabatic effects of the full (parametrized) model. Each potential diabatic source is assigned to a single tracer which then captures the effects of this source on the transport of the PV. In this way the various potential diabatic sources of PV can be identified and their effects quantified adequately. The goal of the current investigation is to highlight the influence of the underlying numerical scheme on the conservation of PV, completely independent of the diabatic effects explicitly accounted for in the physics parametrizations.

As partial motivation for such a comparison, we note that in the discretized equations the smallest scales are truncated and the scale interaction determined by the nonlinear advective term in the momentum equation cannot be imitated by the linear tracer advection equation. Ohkitani (1991) and Babiano and Provenzale (2007) provide a discussion of this problem for incompressible flows in two dimensions. For three-dimensional, fully compressible (even hydrostatic) flow as utilized by most climate models, the passive tracer and dynamical variables are no longer guaranteed to agree once a discrete version of the equations is considered. When certain scales are truncated from the representation of the flow, the inter-scale interaction due to the nonlinear advective term in the momentum equation is not adequately captured, and so the discrete system will no longer maintain consistency, even in the ideal cases of extremely high resolution.

In addition, because the rather coarse typical grid spacings in climate models ( $\Delta x \approx 100-200 \mathrm{~km}$ in the horizontal directions) do not capture all of the physically relevant dissipation scales, a dissipative mechanism is necessary in GCMs to prevent the build-up of kinetic energy and enstrophy that otherwise could accumulate at the smallest, under-resolved grid scales. Kent et al. (2012b) provide a discussion of this with respect to the dynamical cores in CAM which are also used in this study. The dissipative schemes can take the form of filters, explicitly added or implicitly induced numerical diffusion as reviewed in Jablonowski and Williamson (2011). Some of these dissipative mechanisms (particularly linear diffusion) can be well understood, e.g. Whitehead et al. (2011) discussed a particular example of an explicitly added diffusion process in CAM-FV. Some nonlinear dissipative processes though, and their impact on the circulation, are harder to assess although one might 'hope' that their effects are either negligible or truthfully represent the small-scale, unresolvable features of the flow. Frequently the dynamical core uses a different form of dissipation for the tracer transport algorithm and the dynamical integration. The current investigation is an initial step toward quantifying how these differences affect the dynamical core-tracer consistency.

In this study we use the adiabatic baroclinic wave test case described by Jablonowski and Williamson (2006a), primarily because the analytic initial conditions for the dynamic variables allow us to compute exactly an initial condition for the potentia vorticity prescribed as a tracer (tracer PV, see the Appendix). We then follow the tracer during the simulation via Eq. (4), (5) or (6) depending on the model formulation, and compare the tracer's evolution with the computation of the dynamic potential vorticity based on $\mathbf{u}, \theta$, and $\rho$ (dynamic PV). In hydrostatic primitive-equation-based models, only $\mathbf{v}$ and $\theta$ are needed for the computation of the dynamic PV. We propose several methods for measuring the consistency of a model using the tracer PV and dynamic PV as test fields. The techniques are tested within the CAM 5.1 framework (Neale et al., 2010) with its four dynamical cores: CAM-FV, CAM-SE (spectral element), CAM-EUL, and CAM-SLD. We emphasize that the parameter sets and horizontal resolutions we select for each dynamical core are the same used for operational climate simulations, i.e. parameters like the time step or the horizontal diffusion coefficient are chosen to coincide with that used in full-physics model runs to highlight the consistency of the models at climate resolutions. However, we halved the default CAM 5.1 vertical grid spacing which allows very accurate computations of the dynamic PV field.

This article is organized as follows. Section 2 briefly surveys the Jablonowski and Williamson (2006a) baroclinic wave test case in the context of comparing the tracer and dynamic PV, before defining some quantitative measures of model consistency. Section 3 provides limited descriptions of the four CAM 5.1 dynamical cores, and describes the results of the PV consistency assessments. Section 4 discusses the possible implications of this work, and includes suggestions for further work. The equations for the analytic tracer PV initialization are included in the Appendix. These results (and particularly the details in the Appendix) are meant to encourage other modellers to utilize the identical set-up to examine the consistency of their dynamical cores and tracer advection schemes.

\section{Potential vorticity in an idealized setting}

\subsection{Tracer and dynamic PV in an idealized baroclinic wave}

For the current evaluation of model consistency, we consider the adiabatic baroclinic wave test case described in Jablonowski and Williamson (2006a, 2006b). This test case starts with an analytically prescribed balanced initial state with an overlaid small-amplitude zonal wind perturbation placed in the northern midlatitudes. A baroclinic wave develops from this perturbation in the Northern Hemisphere after 4 days of integration, breaking around day 9 at low-lying levels. This provides an ideal situation to consider both the development of linear, yet realistic flow prior to the wave breaking, and nonlinear, multi-scale flow afterward.

Figure 1 illustrates the evolution of the dynamic PV field at the interpolated isentropic 300 and $315 \mathrm{~K}$ levels in a CAM-FV $1^{\circ} \times 1^{\circ}$ simulation with 60 vertical levels and a model top around $2 \mathrm{hPa}$. This resolution corresponds to a horizontal grid spacing of about $110 \mathrm{~km}$ near the Equator. The sloping (with respect to the height or pressure position) $300 \mathrm{~K}$ isentropic level depicted in the left column lies around $800-600 \mathrm{hPa}$ in the region between 40 and $50^{\circ} \mathrm{N}$ and intersects the surface at around $30^{\circ}$, as indicated in white. The $315 \mathrm{~K}$ isentropic level lies at about $550-400 \mathrm{hPa}$ in midlatitudes and does not intersect the ground during the life cycle of the wave considered here. Because this level does not intersect the surface, it is relied on heavily for the analysis performed in the following sections.

Figure 1 highlights the progression and the overturning (breaking) of the baroclinic wave during its rapid development phase between days 6-9. The onset of the breaking and the sharpening of the PV gradients are most distinct at low-lying levels around day 9, but are also evident at higher levels at later times. The wave breaking is graphically identified at the lower level between days 8 and 9, as seen in the left column where the wave starts to fold onto itself. It can also be seen in other fields such as the $850 \mathrm{hPa}$ temperature as displayed in Jablonowski and Williamson (2006a). After the breaking occurs, small-scale structures develop. The appearance of small scales from the mean flow indicates that nonlinear effects become dominant in this region (from $90^{\circ} \mathrm{E}$ to $150^{\circ} \mathrm{W}$ in longitude). We make the distinction between the linear (prior to wave breaking at day 8 ) and nonlinear flow (from day 8 and onward), because the tracer advection algorithm will always be integrating the linear transport equation (4), (5) or (6) for the passive tracer $q$. The dynamical integration is also effectively integrating the transport equation, but now $q=q(\mathbf{v})$ depends on the velocity $\mathbf{v}$ and is thereby an active tracer so that the dynamical evolution is nonlinear. This allows additional discretization inconsistencies to occur as discussed above. 
(a)

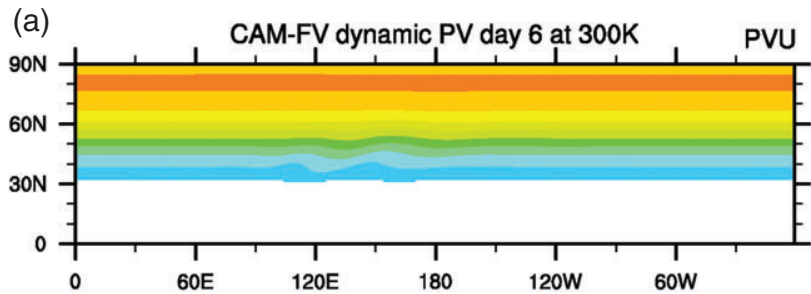

(b)
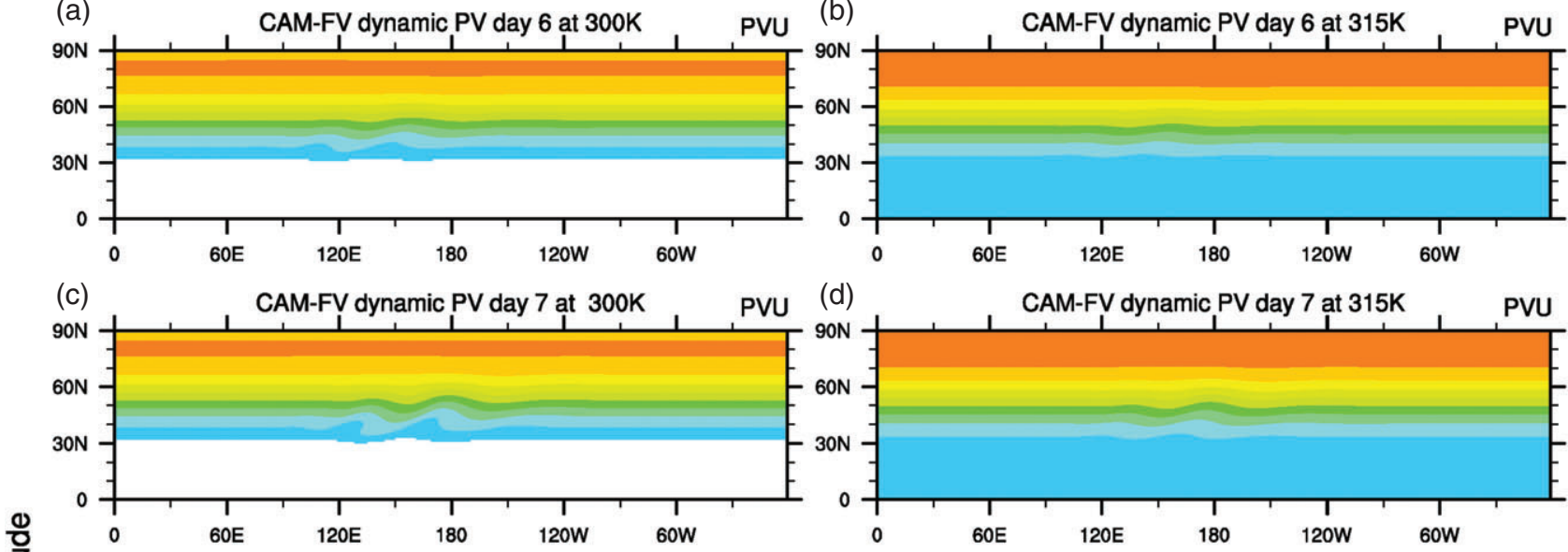

(d)
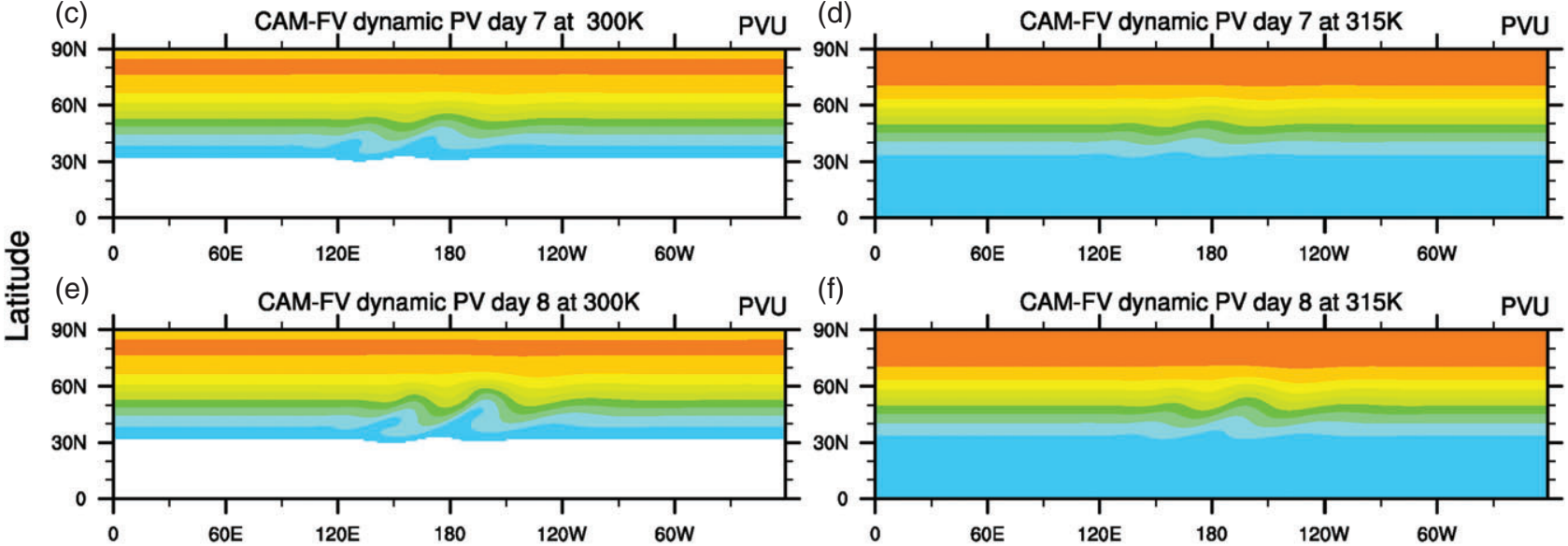

(f)
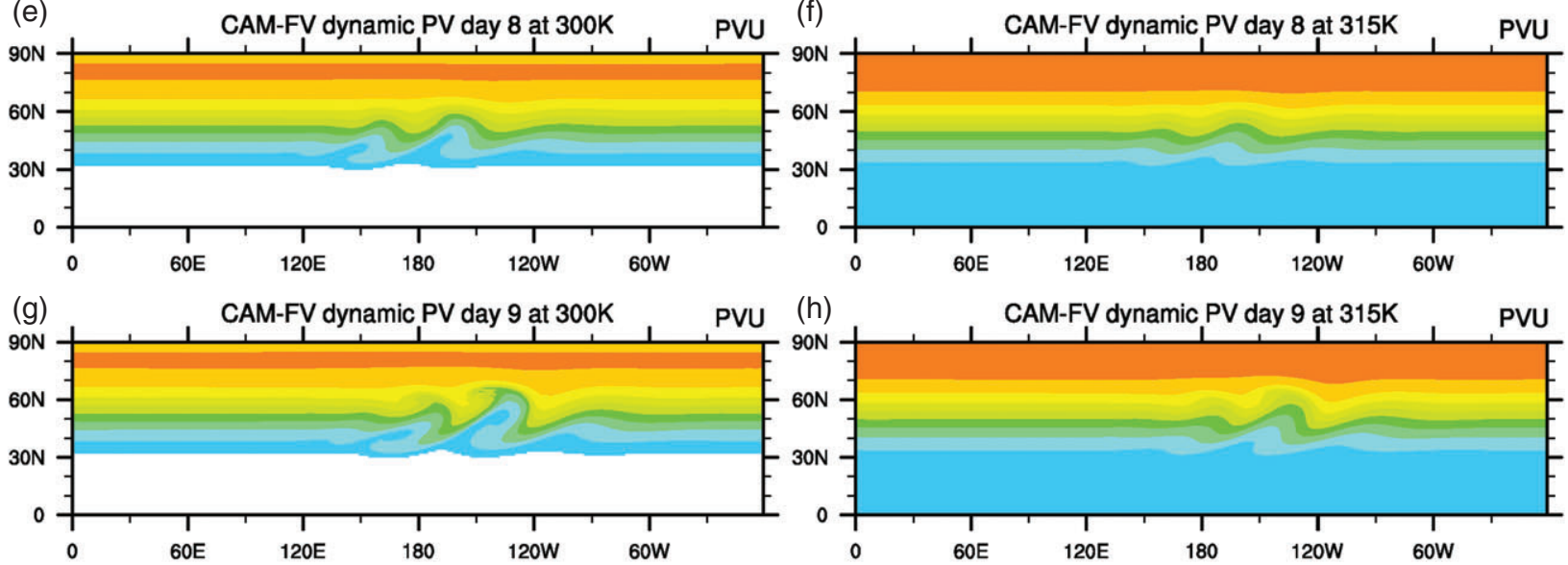

(h)

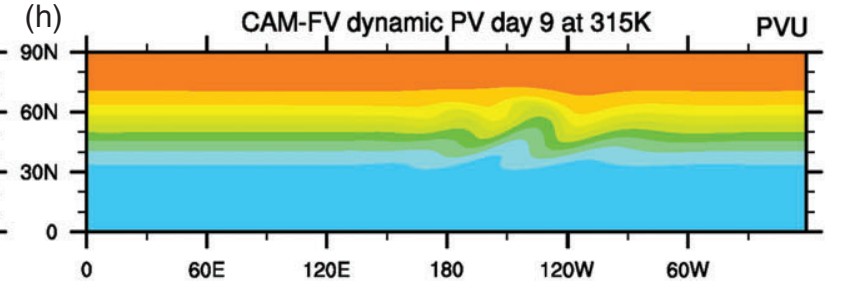

Longitude

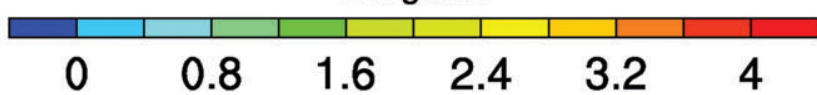

Figure 1. Longitude-latitude cross-section of the dynamic potential vorticity in the Northern Hemisphere as simulated with CAM-FV at the horizontal resolution $1^{\circ} \times 1^{\circ}$ with 60 levels. The PV evolution at days $6,7,8$ and 9 at (a, c,e,g) the $300 \mathrm{~K}$ isentropic level and (b, d, f, h) the $315 \mathrm{~K}$ isentropic level is shown in potential vorticity units (PVU) defined as PVU $=10^{-6} \mathrm{~K} \mathrm{~m}^{2} \mathrm{~kg}^{-1} \mathrm{~s}^{-1}$. The white space at the $300 \mathrm{~K}$ isentropic level indicates where the isentrope intersects the Earth's surface.

We note that the baroclinic wave affects, almost exclusively, a confined latitudinal strip in the Northern Hemisphere over the course of the first 15 days. Therefore, we simplify the comparisons between the dynamic and tracer PV fields, and focus the analyses on the development of the wave in the latitudinal strip between 30 and $90^{\circ} \mathrm{N}$ (unless specified otherwise). In all our analyses the dynamic PV is first computed along CAM's hybrid $\eta$ model levels (Simmons and Burridge, 1981) before being interpolated to the isentropic $315 \mathrm{~K}$ layer. The $315 \mathrm{~K}$ level is not close to the domain boundaries in the mid- and high latitudes and thereby avoids the degradation of the computation due to boundary effects, e.g. the intersection with the surface.

\subsection{Quantitative measures of consistency}

\subsubsection{Paradigms of consistency}

There are two basic premises for quantifying the consistency between dynamics and tracer transport of a model. The first premise involves point to point comparisons, i.e. when comparing two datasets it is assumed that both lie on the same grid so that error norms or scatter plots (as explained in the following subsection) can be determined exactly. This is a valid assumption provided the tracer PV and dynamic PV are compared within the same model framework, and at the same resolutions. It is also a viable approach if the dynamic and tracer PV of the same model experiment are vertically interpolated to isentropic levels via an identical algorithm. The second premise is that data on differing grids must be compared, and the influence of the interpolation methods should be minimized. This is of particular interest when the flow is nonlinear, because errors due to interpolations become more significant in complex flow fields with sharp gradients. An accurate computation of the dynamic PV and the choice of the interpolation method from one grid type or resolution are important for PV assessments. This is especially true in the presence of differing vertical discretizations and grids as discussed in Ziv and Alpert (1994).

\subsubsection{Point to point comparisons: error norms and scatter plots}

A metric used in the analysis of numerical techniques is the discrete $l^{p}$ norm of the error from an exact solution, i.e. if the model data are represented by $q$ with an exact solution corresponding to $q_{\mathrm{T}}$, then

$$
l^{p}\{q\}=\left\{\frac{I\left[\left(q-q_{\mathrm{T}}\right)^{p}\right]}{I\left[\left(q_{\mathrm{T}}\right)^{p}\right]}\right\}^{1 / p}
$$

defines the normalized $l^{p}$ error of $q$ where $I[\cdot]$ denotes the areaweighted global integral (in this case, actually the integral over the latitudinal strip from 30 to $90^{\circ} \mathrm{N}$ at the interpolated isentropic level of $315 \mathrm{~K}$ ) of the given quantity. The normalized maximum $\operatorname{norm} p=\infty$ is

$$
l^{\infty}\{q\}=\frac{\max \left|q-q_{\mathrm{T}}\right|}{\max \left|q_{\mathrm{T}}\right|} .
$$

There is no exact solution known for the baroclinic wave test, but the difference between the tracer PV and dynamic PV is a measure 
of the lack of consistency in the model, i.e. we let $q$ be the tracer PV and $q_{\mathrm{T}}$ the dynamic $\mathrm{PV}$, although this by no means indicates that the dynamic PV is an exact solution. A perfectly consistent model would have identical distributions of tracer PV and dynamic PV. Hence the $l^{p}$ consistency norm will be due to numerical errors and differences in the integration of the dynamics and the tracer advection algorithm.

Traditionally $p=2$ (least squares regression) or $p=\infty$ (maximum error norm) are used for measurements of model error. The $l^{2}$ error norm does not adequately capture the detrimental effects of extreme differences on small scales, and the maximum error norm $l^{\infty}$ weighs these statistically rare events more than desired. Hence we choose to consider the $l^{4}$ norm (an interpolant between $l^{2}$ and $l^{\infty}$ ) of the difference between the dynamic and tracer PV. This provides an accurate measure of the overall error as provided by $l^{2}$ but includes the effect of localized errors provided by $l^{\infty}$. If we consider the distribution defined as the pointwise difference between the dynamic and tracer PV, then the $l^{4}$ norm gives a measure of the kurtosis of this distribution, indicating the tendency of the differences between dynamics and tracers to originate from localized regions as opposed to a global offset.

The $l^{4}$ consistency error norm gives a useful metric for comparing the relative consistency of various models, but it does not indicate the source of these inconsistencies. A qualitative measure related to the consistency error norms are scatter plots such as that illustrated in Figure 2. (Zapotocny et al., 1996, show the use of similar plots.) Each grid point in the domain has both a tracer PV and a dynamic PV value. The horizontal axis of the scatter plot corresponds to the value of the dynamic PV, and the vertical axis is the tracer PV at the same grid point. Ideally the dynamic and tracer PV should agree exactly at all points on the grid, so the scatter plot should follow the line $y=x$ exactly. Deviations from the line $y=x$ then indicate inconsistencies between the dynamic integration and tracer advection algorithm. While it is more difficult to ascertain temporal dependence of inconsistencies with this type of comparison, scatter plots do yield more information than simply calculating the error norm at a given time. For example, as demonstrated schematically in Figure 2 it appears that for smaller values the tracer PV is larger than the dynamic PV, and the opposite is true for larger values of PV. This gives far more insight into the errors in consistency than the simple calculation of an error norm.

For the current investigation, we construct scatter plots for the tracer PV-dynamic PV comparison for days 1, 8, 12, and 15. The traditional PV unit PVU $=10^{-6} \mathrm{~K} \mathrm{~m}^{2} \mathrm{~kg}^{-1} \mathrm{~s}^{-1}$ is used for both axes. This choice of time-stamps will highlight the inconsistency that arises both from the linear flow up to day 8 (in which case the

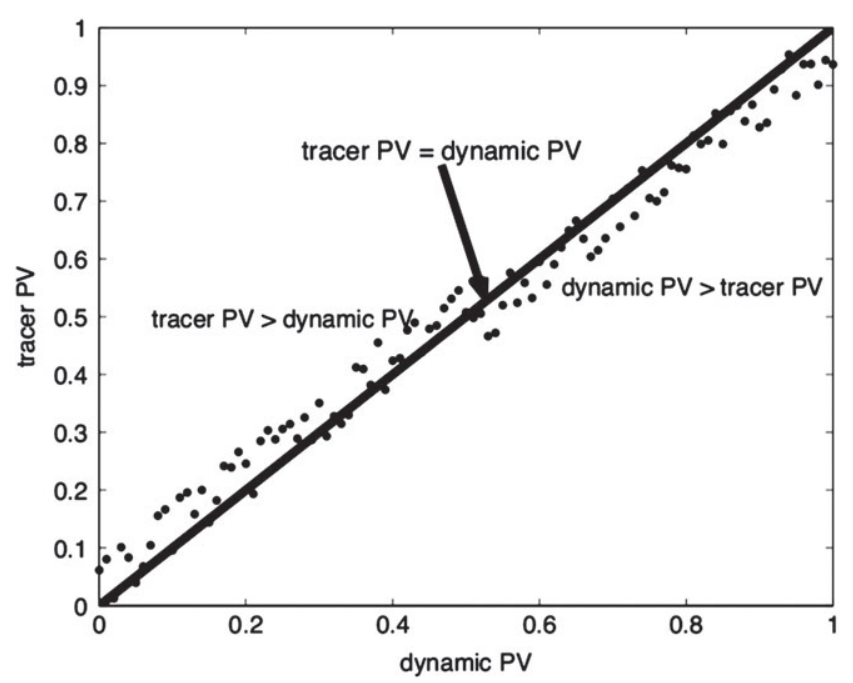

Figure 2. A schematic rendering of a scatter plot comparing dynamic and tracer PV (units: PVU). dynamics and tracer PV should agree very well) and the effects that the nonlinear evolution of the baroclinic wave has on the consistency for days 12 and 15.

\subsubsection{Probability density functions and contour plots}

Theoretically since PV is conserved on closed isentropic surfaces (isentropes that do not intersect the surface of the Earth), the probability density function ( $p d f$ ) describing the distribution of $\mathrm{PV}$ on that surface will not change with time. This provides another useful metric to gauge the conservation/consistency of PV. For the evolution of the baroclinic wave considered in this article (at least up to day 15), there is little to no exchange of PV across the Equator. Assuming such an exchange is negligible, we restrict our attention to the Northern Hemisphere, meaning that a pdf constructed from either the tracer or dynamic PV for each model should not only be the same at any instant of time, but should not evolve with time, i.e. the dynamic and tracer PV pdf should be the same at all times on the selected closed isentropic surface ( $315 \mathrm{~K}$ in the Northern Hemisphere).

For the results reported in section 3, we construct a pdf for both the dynamic and tracer PV interpolated to $315 \mathrm{~K}$ in the Northern Hemisphere by binning the PV into bins of size $\beta$. For the results discussed in section 3 , the bin size is subjectively chosen as $\beta=0.2$ PVU. Rather than considering the temporal evolution of the dynamic and tracer pdfs for each dynamical core, we consider the difference in these pdfs from the original pdf of the initial state. This indicates the departure of the distribution from what should be a conserved shape, and makes the differences easier to recognize. Recognizing that the distributions in the linear flow regime are nearly identical to the initial state, we consider these differences in the pdfs only at day 15.

Finally, although all of the metrics discussed thus far provide quantitative information with respect to the consistency of a given dynamical core, it is important to also evaluate differences in the fields themselves. In the interest of considering the evolution of the PV only on isentropic surfaces that do not intersect the surface boundary, we look at contour plots of the dynamic and tracer PV at $315 \mathrm{~K}$. Section 3 displays such a comparison only at day 15 as the differences between tracer and dynamic PV are the most stark at this point, although it is instructive to consider the complete temporal evolution of both quantities on this isentrope.

\section{Consistency in CAM 5.1}

The versatility of CAM's framework is displayed in the work of Rasch et al. (2006) wherein three dynamical cores are compared, while using the same physics package. We take a similar approach, although we assess the consistency of each of the four dynamical cores in CAM version 5.1 without any physics parametrizations. Each configuration and its parameter set outlined below represents a default which would typically be used at climate resolutions with $100-160 \mathrm{~km}$ grid spacing near the Equator. We thereby mimic realistic simulations once parametrized physics are included. We make no attempt to match the parameters, like the tracer and dynamics time steps or diffusion mechanisms, from one model to another and note that these are dependent on the numerical schemes, their stability characteristics and the horizontal grid spacings. A detailed parameter sensitivity study, although highly desirable, is beyond the scope of this article.

\subsection{Model descriptions}

Before examining the results for each of these dynamical cores, we first give a brief description of each. Particular emphasis is placed on the subgrid dissipative mechanisms (filters, diffusion) that are added to each dynamical core. We do not explore every possible form of dissipation or mixing processes in CAM, but consider a few key points that may illustrate differences in the consistency of each model. 


\subsection{1. $C A M-F V$}

The finite volume dynamical core (CAM-FV) and its corresponding tracer transport algorithm are both based on the flux-form semi-Lagrangian scheme of Lin and Rood (1996) on the latitude-longitude grid. The method is a dimensional splitting technique that relies on the one-dimensional finite volume methods akin to the van Leer-type monotonic methods (van Leer, 1974, 1977) or the Piecewise Parabolic Method (PPM; Colella and Woodward, 1984). The extension of this tracer advection algorithm to the shallow-water equations was carried out in Lin and Rood (1997) with further application to three-dimensional hydrostatic motion introduced in Lin (2004). The vertical discretization is based upon a floating Lagrangian approach with periodic remapping to a reference grid. In our study the flow is allowed to freely evolve within the Lagrangian layers for ten dynamics time steps before remapping is invoked.

Implicit diffusion is added to both the dynamics and tracer transport through the nonlinear limiters placed on the underlying one-dimensional finite volume methods. In addition, an explicitly added diffusion mechanism in the form of horizontal divergence damping is implemented in CAM-FV (Whitehead et al., 2011). This explicit damping mechanism is present only in the dynamics; there is no analog in the tracer transport for this model, indicating a possible source of inconsistency. In this article, the fourth-order horizontal divergence damping mechanism is used. A sponge layer is used to absorb upward traveling waves near the model top to lessen wave reflections. This is applied near the model top in the dynamics using low-order numerics and second-order divergence damping with an increased damping coefficient.

\subsubsection{CAM-EUL}

The spectral-transform Eulerian dynamical core (CAM-EUL) utilizes an underlying quadratic Gaussian transform grid which closely resembles a latitude-longitude mesh. The momentum equations are formulated in vorticity-divergence form. Using spherical harmonics, the prognostic variables are then cast into spectral space and integrated forward in time with a three-timelevel leapfrog method. A fourth-order horizontal hyper-diffusion term is added for stability purposes which is applied to the relative vorticity, horizontal divergence and temperature fields. In addition, second-order horizontal diffusion is activated near the model top to mimic a three-layer sponge zone with a base coefficient of $2.5 \times 10^{5} \mathrm{~m}^{2} \mathrm{~s}^{-1}$. Additional details on the diffusion and sponge are provided in Jablonowski and Williamson (2011). CAM-EUL uses different methods for the advection of tracers and the evolution of the dynamics. In particular, it employs a monotonic semi-Lagrangian tracer advection scheme that is dimensionally split in the horizontal and vertical directions. Both the interpolations in the semi-Lagrangian scheme and the monotonic constraint are sources of implicit numerical diffusion for the tracers. In addition, the dynamical core and the tracer advection scheme in CAM-EUL invoke a 'mass-fixer' to ensure that conservation of dry air mass and tracer mass is achieved.

\subsubsection{CAM-SLD}

The spectral-transform dynamical core CAM-SLD is based on a semi-Lagrangian approach in both the dynamical core and its tracer advection algorithm, and utilizes the same Gaussian grid as CAM-EUL. However, the semi-Lagrangian interpolations in the tracer advection scheme are monotonic (shape-preserving) whereas they remain unconstrained in the dynamical core. As in CAM-EUL, CAM-SLD is not mass-conserving by design and utilizes a mass fixer. In contrast to CAM-EUL, the tracer transport in SLD is performed in a single three-dimensional step with no dimensional splitting, and SLD utilizes the horizontal velocities $u$ and $v$ as prognostic variables. The time integration is based on a two-time-level semi-Lagrangian semi-implicit time-stepping mechanism. To damp dispersive errors inherent to the spectraltransform spatial discretization, a fourth-order hyper-diffusion term is included in the dynamic calculation, in addition to a second-order diffusive three-layer sponge zone near the model top.

\subsubsection{CAM-SE}

The spectral element (SE) component of CAM (Taylor and Fournier, 2010; Taylor, 2011) is built on the cubed sphere grid to avoid the singularities generated by a latitude-longitude grid near the poles and increase the scalability on high performance computing platforms (Dennis et al., 2005, 2012). CAM-SE utilizes the spectral element approach developed initially for the shallowwater equations in Taylor et al. (1997) and later expanded to the hydrostatic atmosphere (Neale et al., 2010, gives further references). The runs in this article use a third-order polynomial reconstruction in each element (which is fourth-order accurate). This choice is typical for operational runs. The dynamics and tracer transport are treated similarly in CAM-SE with the tracer transport employing a quasi-monotone limiter (Taylor et al., 2009) which renders the tracer advection scheme second-order accurate. However, note that the Runge-Kutta time-stepping schemes are slightly different in the dynamical core and the tracer transport scheme, and that different vertical discretizations are used. The CAM-SE dynamical core makes use of a finite-difference scheme in the vertical, whereas CAM-SE's tracer advection scheme utilizes a flow-following Lagrangian coordinate with periodic vertical remapping to a reference grid as in CAM-FV. An explicit fourth-order horizontal hyper-diffusion with identical diffusion coefficients is added to both the dynamics and tracer advection to maintain stability. In addition, the dynamical core applies a second-order horizontal diffusion near the model top to damp the reflection of upward travelling waves.

\subsection{Model comparisons}

Table 1 details the model configurations for each of the dynamical cores. As noted before, the diffusion parameters and time steps are the default values for these resolutions, individually tuned for each dynamical core so that the climate simulations of the full-physics model will yield realistic results. All comparisons are done at climate-type horizontal resolutions which are T85 (triangular truncation) in case of CAM-EUL and CAM-SLD and approximately $1^{\circ} \times 1^{\circ}$ in case of CAM-FV and CAM-SE. These resolutions correspond to horizontal grid spacings between 100 and $160 \mathrm{~km}$ near the Equator and highlight the effects that unresolved subgrid processes have on the consistency between dynamics and tracers. The T85 and $1^{\circ} \times 1^{\circ}$ resolutions can also be considered 'equivalent' as determined via aqua-planet studies by Williamson (2008), despite their differences in the number of total grid points. Each model was run with 60 vertical levels (L60). This vertical resolution halves the vertical grid spacings that are typically used in CAM5. The hybrid coefficients for the default 30-level (L30) configuration are listed in Reed and Jablonowski (2012) which enables other modelling groups to use an identical set-up. We chose the higher vertical resolution over CAM5's standard 30 levels in order to compute vertical derivatives in the calculation of the dynamic PV more accurately. However, we also conducted L30 experiments (not shown) which led to identical conclusions.

\subsubsection{Point to point comparisons: Error norms, scatter plots and extreme values indicated by contour plots}

A plot of the normalized percentage $l^{4}$ error for CAM-FV, CAMEUL, CAM-SLD, and CAM-SE is shown in Figure 3 for the model configurations detailed in Table 1. The initial differences between the dynamic PV and tracer PV as indicated by the errors at day 0 (Figure 3(a)) show that the discrete algorithm for calculating 
Table 1. Horizontal model resolutions, time steps $\Delta t$ for both the dynamics and tracer transport and horizontal fourth-order hyperdiffusion coefficients. The triangular truncation T85 is the highest resolved wavenumber in CAM-EUL and CAM-SLD. The CAM-SE resolution ne30np4 describes that each cubed-sphere face is divided into $30 \times 30$ elements with additional $4 \times 4$ collocation points per element to support a third-order polynomial. CAM-FV applies fourth-order horizontal divergence damping as explained in Whitehead et al. (2011).

\begin{tabular}{lcccr}
\hline $\begin{array}{l}\text { Dynamical } \\
\text { core }\end{array}$ & $\begin{array}{c}\text { Horizontal } \\
\text { resolution }\end{array}$ & $\begin{array}{c}\text { No. of horizontal } \\
\text { grid points }\end{array}$ & $\begin{array}{c}\text { Approx. grid length } \\
\text { at Equator }(\mathrm{km})\end{array}$ & $\begin{array}{l}\text { Dynamics } \Delta t \\
(\text { tracer } \Delta t)(\mathrm{s})\end{array}$ \\
\hline CAM-FV & $1^{\circ} \times 1^{\circ}$ & $181 \times 360$ & 110 & $\begin{array}{c}\text { Diffusion coefficient } \\
\left(\times 10^{15} \mathrm{~m}^{4} \mathrm{~s}^{-1}\right)\end{array}$ \\
CAM-EUL & T85 & $128 \times 256$ & 156 & - \\
CAM-SLD & T85 & $128 \times 256$ & 156 & $600(600)$ \\
CAM-SE & ne30np4 & $6 \times 30 \times 30$ elements & 110 & $1800(1800)$ \\
\hline
\end{tabular}
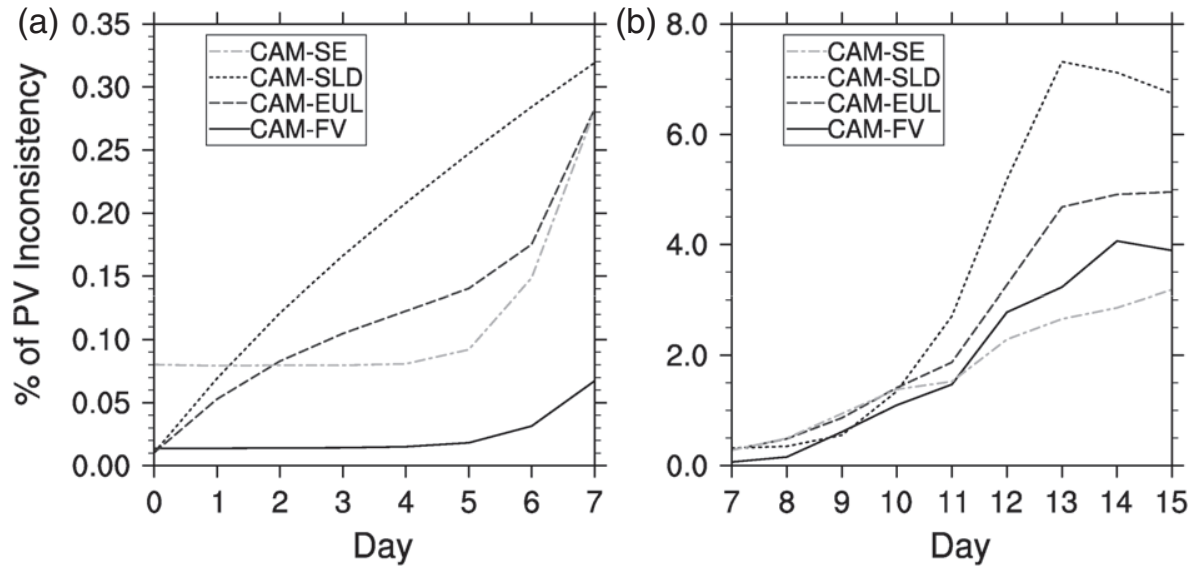

Figure 3. Evolution of the percentage of the nomalized $l^{4}$ norm of the difference between dynamic and tracer PV for all four dynamical cores. (a) shows the linear flow during the first 7 days of the test case, and (b) the evolution of the consistency error during the transition from linear to nonlinear flow (days $7-9$ approximately), and the evolution of inconsistency for the fully developed nonlinear regime (days 9-15). We consider the PV interpolated to $315 \mathrm{~K}$ contained in the region between $30^{\circ} \mathrm{N}$ and $90^{\circ} \mathrm{N}$. Note the difference in scale for the vertical axes.

dynamic PV seems to penalize the CAM-SE model relative to the other three dynamical cores. CAM-SE's initial errors are likely due to interpolations of the output from the cubed-sphere to the regular latitude-longitude grid (thus simplifying the discrete computation of dynamic PV). Across all four dynamical cores, the maximum initial inconsistency is small and less than $0.1 \%$. Cubic splines were used for the computation of the vertical potential temperature gradient that is part of the PV formulation. A centred finite-difference type method is discouraged for the computation of the potential temperature gradient since it was found to be less accurate.

When the flow is linear with smooth PV distributions at the $315 \mathrm{~K}$ isentropic level, all four dynamical cores are quite consistent, with consistency errors well below $0.5 \%$ even up to day 7 (Figure 3(a)). As the flow develops more fully (Figure 3(b)), the consistency error worsens, with all four models showing an approximately linear growth in the error until day 11. Around days 11 and 12 though, the spectral-transform models (CAM-EUL and CAM-SLD) demonstrate a significant departure from consistency. As described earlier, the spectral-transform dynamical cores are built on similar premises, so the concurrent development of inconsistency at this point may be an indication that the errors are linked to the spectral-transform technique (e.g. occurrence of Gibb's oscillations, generation of small-scale noise or dispersion errors). The dissipative effect of the semiLagrangian interpolations in CAM-SLD might have also become more prominent after day 11 as they are triggered by smallscale gradients in the flow field. It is worth noting that CAM-SE outperforms the other three dynamical cores in this measurement of consistency, even though the initial inconsistency is worse (at day 0 due to interpolation errors). The better consistency might be a result of CAM-SE's relatively high-order yet local numerical scheme.

Scatter plots analyzing the consistency of each dynamical core at days 1, 8, 12, and 15 are shown in Figures 4-7. One feature that is highlighted in all four of these scatter plots is that the tracer $\mathrm{PV}$ appears to be bounded by its initial range, i.e. the maximum
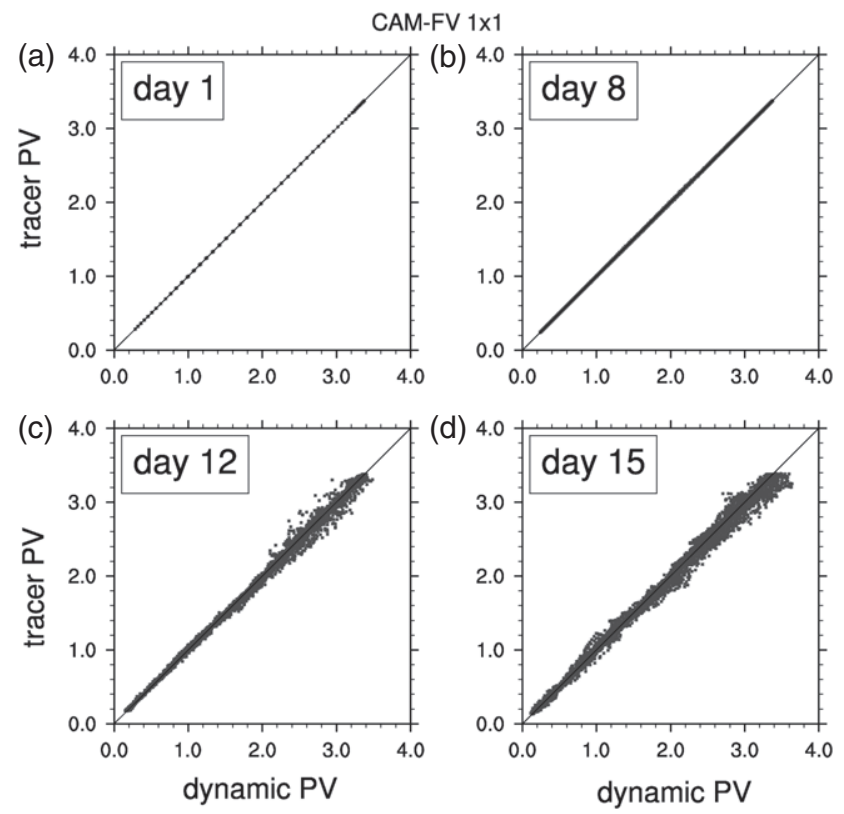

Figure 4. Scatter plots of the tracer and dynamic PV (PVU) at (a, b, c, d) days $1,8,12$ and 15 for a CAM-FV $1^{\circ} \times 1^{\circ}$ L60 simulation in the region $30-90^{\circ} \mathrm{N}$ at the $315 \mathrm{~K}$ isentropic level. Any deviations from the line $y=x$ indicate differences between tracer PV and dynamic PV.

tracer PV at day 1 is the same as the maximum tracer PV value at day 15. In contrast to the tracer PV, the dynamic PV of CAM-FV, CAM-EUL, and CAM-SLD all have values at day 15 that exceed the initial dynamic PV values. This indicates that the integration of the dynamic PV in these three models is not monotonic, i.e. spurious extrema can be introduced, or physical extrema can be enhanced beyond their physically realistic values. On the other hand, CAM-SE does well in this aspect and appears to retain the same maximal values of dynamic PV that are present at day 1. 

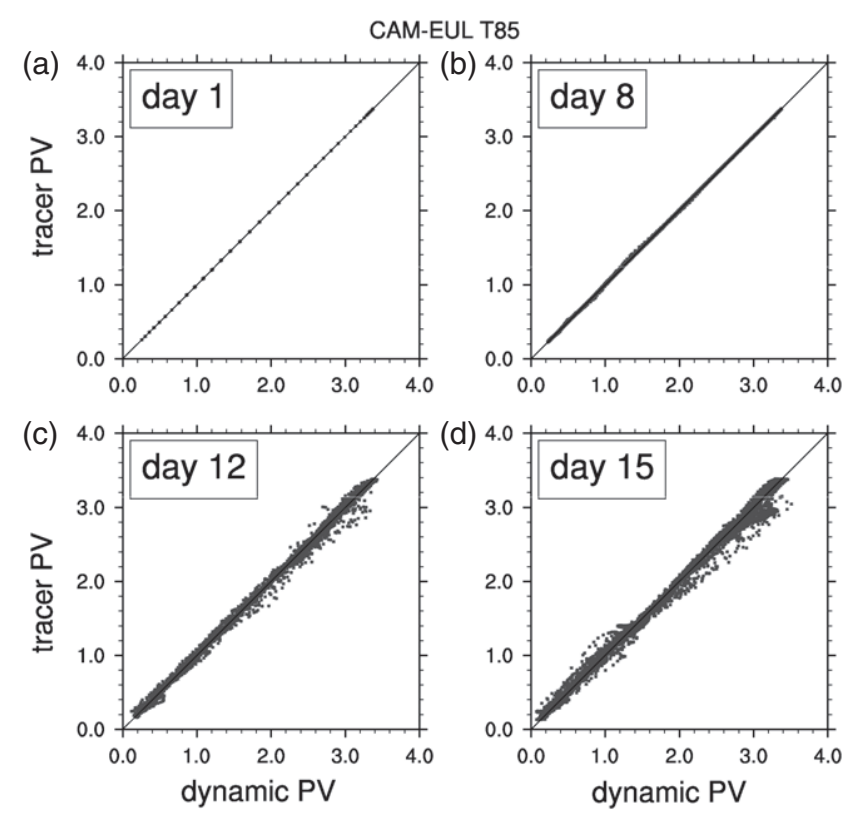

Figure 5. As Figure 4 but for CAM-EUL T85L60.
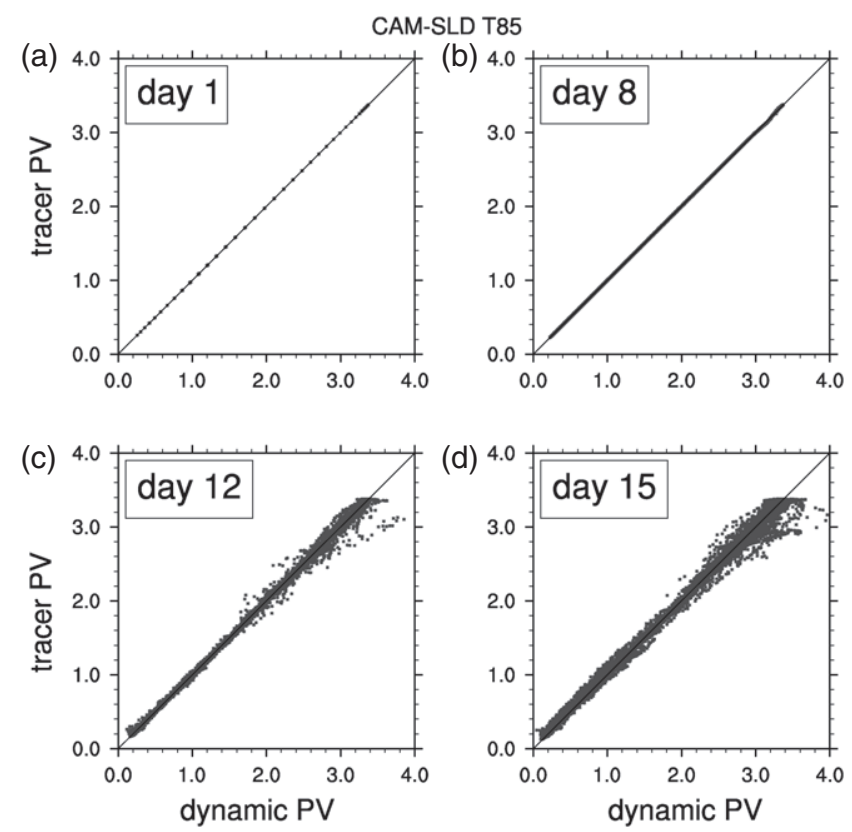

Figure 6. As Figure 4 but for CAM-SLD T85L60.

CAM-SE's preservation of the maximum dynamic PV accounts for some of the better consistency observed in Figure 3.

Another observation in conjunction with that noted in Figure 3 is that all four models are very consistent in the linear flow regime, as evidenced by the top two plots in each of Figures $4-7$. Once the wave breaks and the flow develops into the nonlinear regime, the dynamics and tracer separate as evidenced by departures from the 1:1 line (tracer PV = dynamic PV). These inconsistency errors appear to depend on the values of the PV.

At day 12, CAM-FV has developed some spread about the 1:1 line. Moderate values of the dynamic PV (between 1.5 and 2.0 PVU) generally correspond to equal or lower values of tracer PV. Departures from consistency in the smaller values of PV (less than 1.5 PVU) are less significant and appear to have no preference toward larger values of either the tracer or dynamic PV. Similar statements can be made for the two spectral-transform dynamical cores, where the departures from consistency are more noticeable especially at day 15. In particular, both CAM-EUL and CAM-SLD seem to bias toward large tracer PV for PV less than 1.5 PVU. This same trend is apparent for the very largest PV values (greater than 3.0 PVU) for CAM-EUL. CAM-SLD does not exhibit a definitive bias for these largest PV values, but has dynamic PV that has exceeded the initial maximum value of 3.4 PVU. For PV in the moderately high range (from 2.0 to $3.0 \mathrm{PVU}$ ), there seems to be a bias toward larger dynamic PV (lying below the 1:1 line) for the spectral transform models. Although the departures from consistency are not as distinct for CAM-SE, one can see that there is a slight tendency for larger dynamic PV for most of the PV values, with the only significant departure from this observation occurring at the smallest PV values near 0.2 PVU where there is substantially larger tracer PV than dynamic PV.

In concert with Figure 3, we note that these scatter plots indicate that CAM-SE is more consistent than the other three models as indicated by how close the scatter plot at day 15 in Figure 7 is to the 1:1 line. We suggest that the different biases of each model toward larger dynamic or tracer PV are indicative of the different treatments each model has for the tracer and dynamic integrations. The PV differences in CAM-EUL are most likely due to the numerical inconsistencies between the dynamics and tracer transport scheme. As noted previously, the dynamics are treated via a spectral-transform method which may develop dispersive errors that are damped out effectively in the semi-Lagrangian tracer transport used in the same model. Such dispersive errors may also be present in CAM-SLD despite the additional dissipation provided by the (non-shape-preserving) semi-Lagrangian interpolations in the CAM-SLD dynamical core. The dissipation in CAM-SE (at least for the model configuration used here) is primarily due to an explicit fourth-order hyper-diffusion for both the dynamics and tracers, indicating a consistent (between dynamics and tracers) treatment of the subgrid scales, leading to less biases in the scatter plots. CAM-FV treats the subgrid scale in the tracer advection algorithm via one-dimensional limiters that preserve monotonicity in the longitudinal and latitudinal directions. The same finite-volume approach is utilized in the dynamical core, but the dimensional splitting can introduce unphysical overand undershoots more frequently for the nonlinear part of the flow. This is likely to cause the biases observed for CAM-FV in Figure 4.

Figure 8 shows a snapshot of the dynamical and tracer PV at the $315 \mathrm{~K}$ isentropic level at day 15 , the same day as represented in (d) of Figures 4-7. We note first that the tracer PV from all of the models are qualitatively similar. For example, in the region $0-60^{\circ} \mathrm{E}, 55-80^{\circ} \mathrm{N}$, all of the tracer PV plots (Figure $8(\mathrm{~b}, \mathrm{~d}, \mathrm{f}, \mathrm{h})$ ) show two distinct orange maxima (2.8-3.2 PVU). Visual examination of the plots shows that fine filaments (for example the ones pointed out by the green arrows) are present in all simulations.
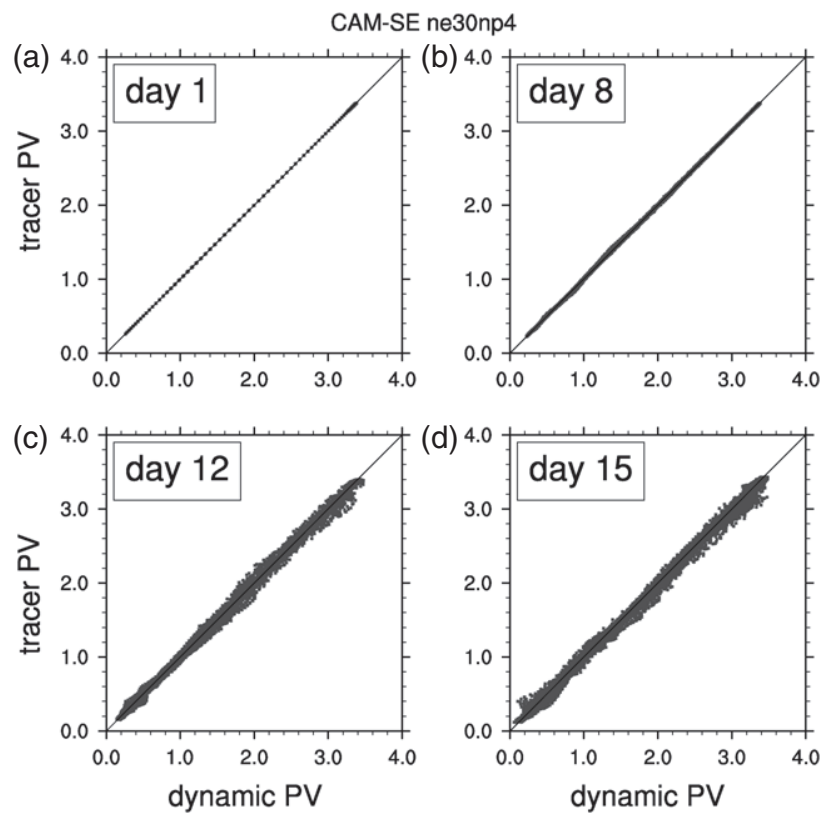

Figure 7. As Figure 4 but for CAM-SE ne30np4 L60. 
(a)

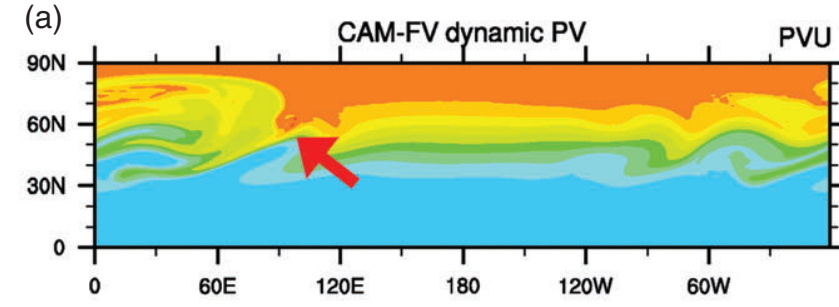

(b)
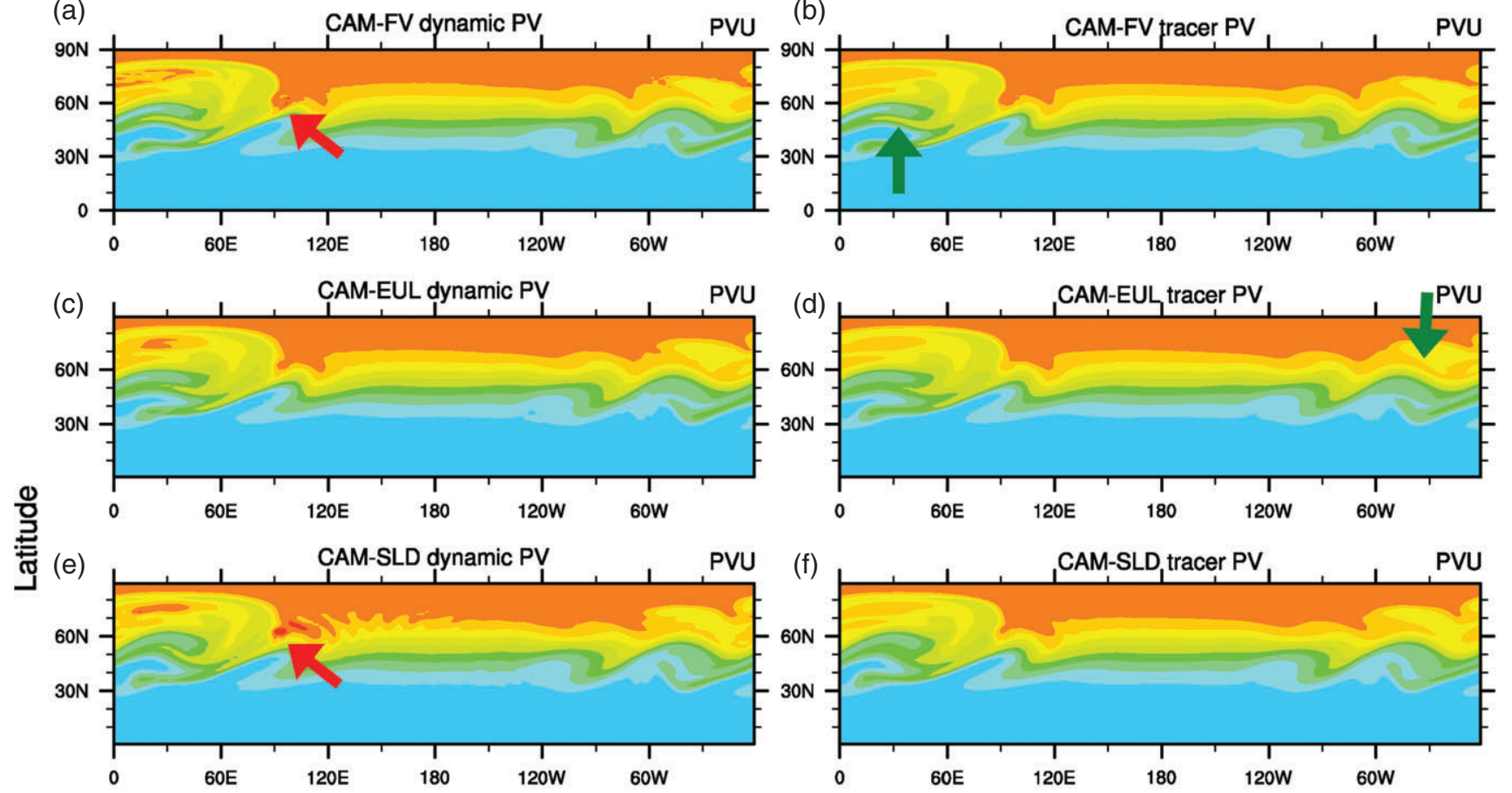

(g)

CAM-SE dynamic PV

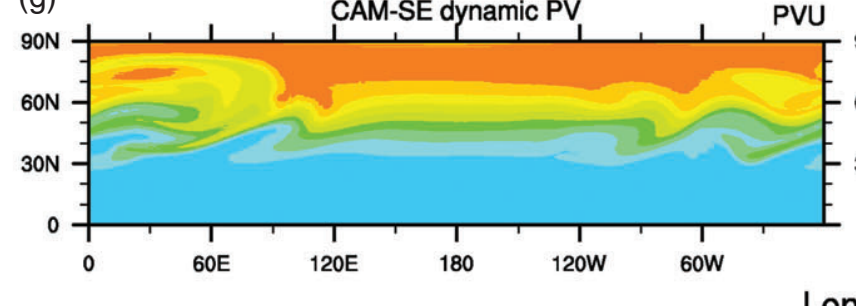

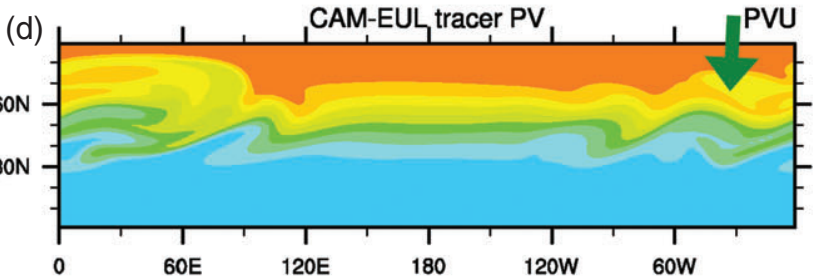

(d)

$\begin{array}{llllll}0 & 60 \mathrm{E} & 120 \mathrm{E} & 180 & 120 \mathrm{~W} & 60 \mathrm{~W}\end{array}$

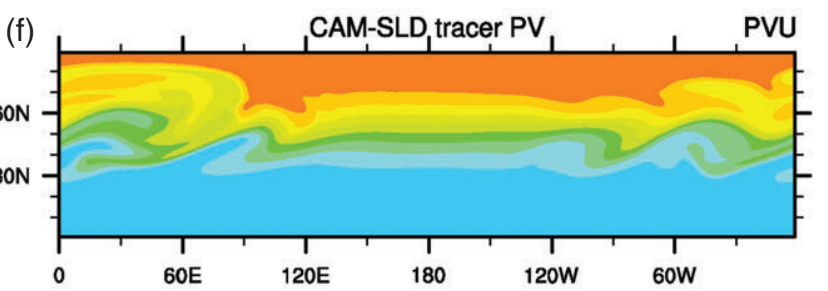

(h)

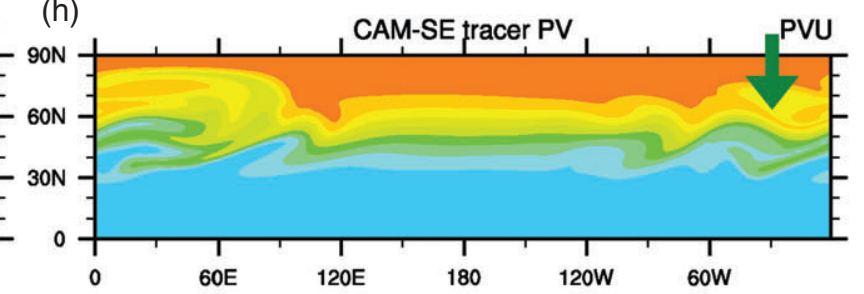

Longitude

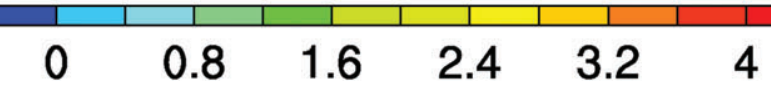

Figure 8. A snapshot at day 15 of the (a, c, e, g) dynamic and (b, d, f, h) tracer PV at the $315 \mathrm{~K}$ isentropic level in the Northern Hemisphere for the four dynamical cores to illustrate the differences in their treatment of the dynamics and tracers. The models are run with the configuration described in Table 1.

However, filaments in one simulation are not present in the same place as the others. There is no clear distinction of the tracer PV from one dynamical core to the next. Simulations at different resolutions (not shown) show that the qualitative structure of the highs and lows and filamentation are sensitive to resolution. The similarity of the tracer PV simulations shown here substantiate the conclusion of Williamson (2008) that we relied on in our choice of equivalent resolutions for the four dynamical cores.

On Figure $8(\mathrm{a}, \mathrm{c}, \mathrm{e}, \mathrm{g})$ are the results for the dynamical PV. Looking again at $0-60^{\circ} \mathrm{E}$ and $55-80^{\circ} \mathrm{N}$, there are discernible differences in the dynamical cores. In all of the dynamical cores there is a maximum exceeding 3.2 PVU. This maximum is not in the tracer PV. In CAM-FV Figure 8(a), there is more smallscale structure in this maximum than in the other dynamical cores. At the location of the red arrow, CAM-FV and CAM-SLD have dynamical PV values which were not present in the initial condition and therefore are unphysical under advection. These maxima are likely the source of the large dynamic PV values noted in Figures 4-7.

Note also to the east of the red arrow in Figure 8(e) the obvious appearance of rippling. Very close examination of the dynamical PV plots show the emergence of small-scale structures that are not present in the tracer PV. This is apparent, for example, in between the darker and lighter blues at $\approx 30^{\circ} \mathrm{N}$. For this choice of model parameters, CAM-SE has the best agreement of the four dynamical cores between tracer PV and dynamic PV.

From these figures we start to see the breakdown of consistency between the tracer PV and the dynamical PV. This appears at scales that are small, and where the dissipative processes in the model are becoming influential. The larger differences are seen in the dynamical PV, where the presence of small-scale structures is amplified in the calculation of the PV. Figure 8 indicates the value of qualitative plots of the PV, allowing the identification of the spatial structure where inconsistencies occur. This may ultimately provide insight into the interplay between numerical methods and the representation of the physics of mixing in climate models.

\subsection{Probability density functions}

Figure 9 shows the difference between the Northern Hemisphere pdfs calculated at day 15 and the initial Northern Hemisphere pdf at day 0 , for the tracer and dynamic PV of the four dynamical cores considered here. Day 15 is shown to display the effect that the fully nonlinear flow can have on the pdfs. Prior to day 15 the magnitude of the difference values do not exceed 0.02 .

The change in the pdfs for the spectral transform dynamical cores has considerably more isolated features (spikes in the Figure) than in the other two dynamical cores. Prior to day 15, the pdfs of CAM-SE and CAM-FV are similar and not as jagged as CAM-EUL and CAM-SLD. The structure of CAM-FV begins to diverge from CAM-SE between days 12 and 15. One feature that all four dynamical cores have is the introduction of a higher probability for PV (both tracer and dynamic) near a PV value of 3.2 PVU. This peak at 3.2 PVU was not present at earlier times, and developed earlier in the spectral transform dynamical cores. In all dynamical cores the structure at PV values greater than 2.0 increases as the flow becomes nonlinear, after day 9 .

For clarification of the higher values consider the features present at $30^{\circ} \mathrm{E}$ and $75^{\circ} \mathrm{N}$ in Figure 8 . This region has $\mathrm{PV}$ of 3.2 PVU. In the dynamical PV the values are higher, and this is 


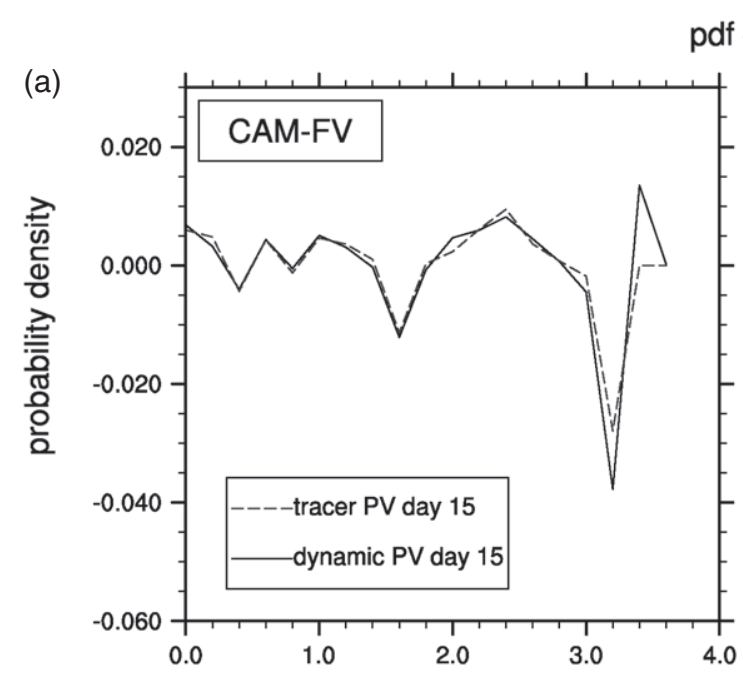

dif difference
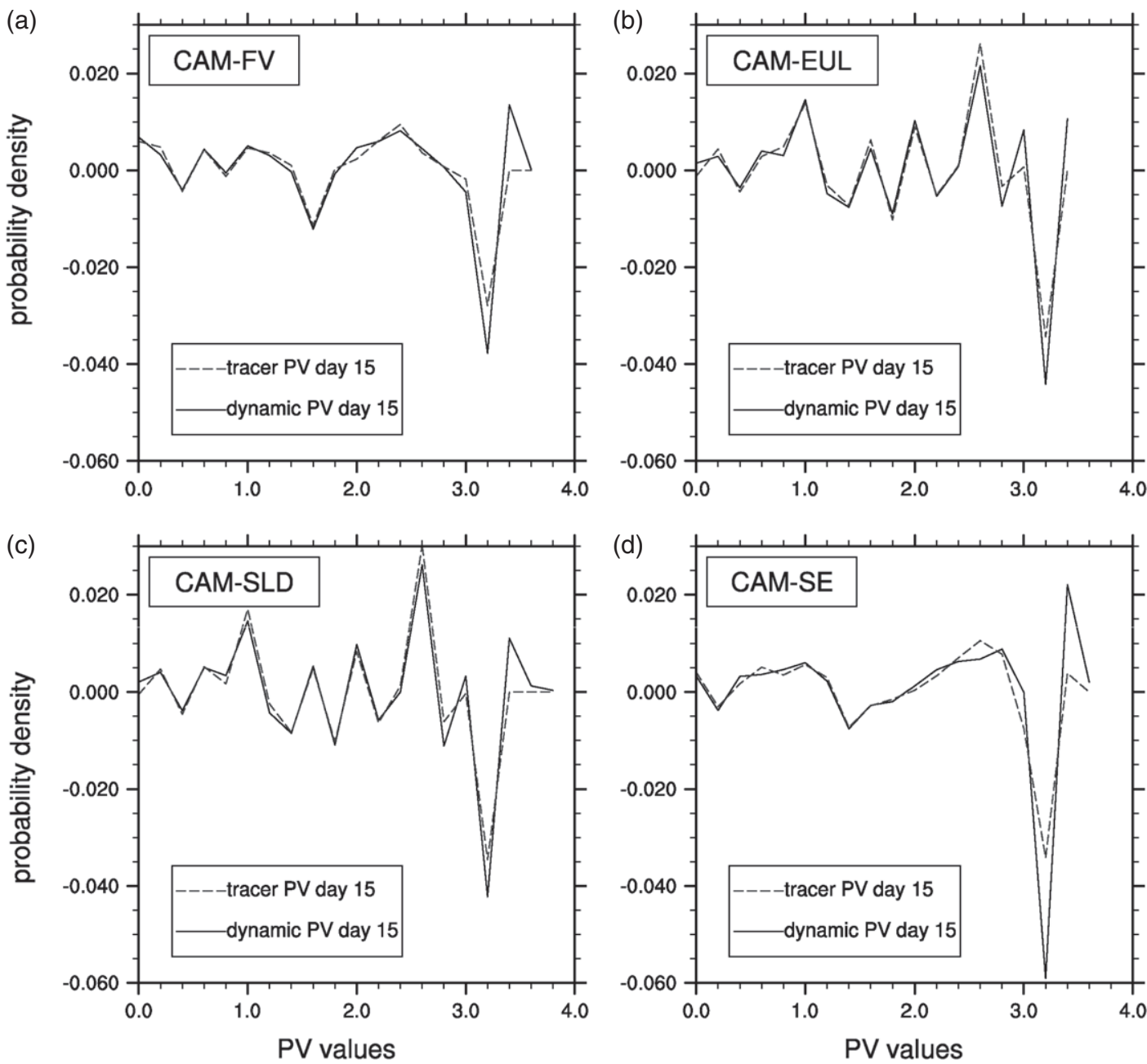

Figure 9. Difference between the Northern Hemisphere probability density functions at day 15 with the initial state for the dynamic and tracer PV at $315 \mathrm{~K}$ for dynamical cores (a) CAM-FV, (b) CAM-EUL, (c) CAM-SLD and (d) CAM-SE, with parameters described in Table 1. The horizontal axis is in PVU.

where the dynamical PV in CAM-FV is starting to show spatial structure indicative of grid-scale noise. These small scales activate the implicit dissipative mechanisms in addition to the explicit methods in use. For these high values, the difference of the pdfs for the tracer PV and the dynamical PV increases. This increase is largest in CAM-SE. At day 15 and later, the very values of PV highlighted by the red arrows in Figure 8 start to become more prominent and suggest that the simulation has reached its predictive end.

\section{Discussion and summary}

We have presented an explicit method for testing the consistency between the representation of dynamical variables and passive tracers in a dynamical core. This was accomplished by including tracer advection of the PV in the baroclinic wave test case of Jablonowski and Williamson (2006a). This demonstrates the usefulness of PV as a diagnostic tool in evaluating the consistency between a model's dynamics and its tracer transport algorithm.

In the Introduction we documented the need for numerical schemes to be consistent in their representation of transport and mixing of mass, momentum and energy. The simulations presented here suggest a relationship between dissipation and consistency which we evaluated more fully using the CAM-FV model. A configuration that has extraordinarily good consistency is to run CAM-FV with upwind (first-order) one-dimensional operators for the dynamics (not shown). This is extremely dissipative, effectively damping the baroclinic wave so much that the wave does not break until well after day 10, whereupon the nonlinear growth is suppressed so the flow relaxes to a laminar state. This leads to a fundamentally different solution which is quasi-linear, allowing the tracer advection algorithm to perform very well. In this case the consistency of the model for any of the possible tracer algorithms in CAM-FV is very good, but the scheme is highly inaccurate. Throughout these simulations this tension between dissipation, consistency and accuracy are realized.

The results obtained here indicate that CAM-SE is the most consistent of the four dynamical cores in CAM. Simulations are sensitive to resolution, and hence effort should be taken to assure that the simulations are equivalent in the sense of Williamson (2008) before comparative conclusions are drawn. Simulations of CAM-SE with a varied diffusion coefficient (not shown) indicate that the consistency of CAM-SE is directly related to diffusion, and the default resolution and diffusion coefficient reported here maintain consistency in a balance with accuracy as good as or better than the other schemes. In addition, we note that our comparisons suggest that some of the differences observed by Rasch et al. (2006) may be explained by the differences in the sub-grid treatment between the different dynamical cores.

The results reported here are for climate resolution studies in which climate-relevant, small-scale features of the nonlinear flow are not fully resolved. We have focused on isentropic surfaces which do not intersect the ground. In all four of the dynamical cores tested here, large inconsistencies develop on isentropes which intersect the surface, as illustrated in Figure 10 at the lowlying $285 \mathrm{~K}$ isentropic level at day 15 . The spurious maximum dynamic PV values (called spurious here because they are not present in the tracer PV) which appear where the isentrope intersects the surface (the red regions especially visible from 0 to 
(a)
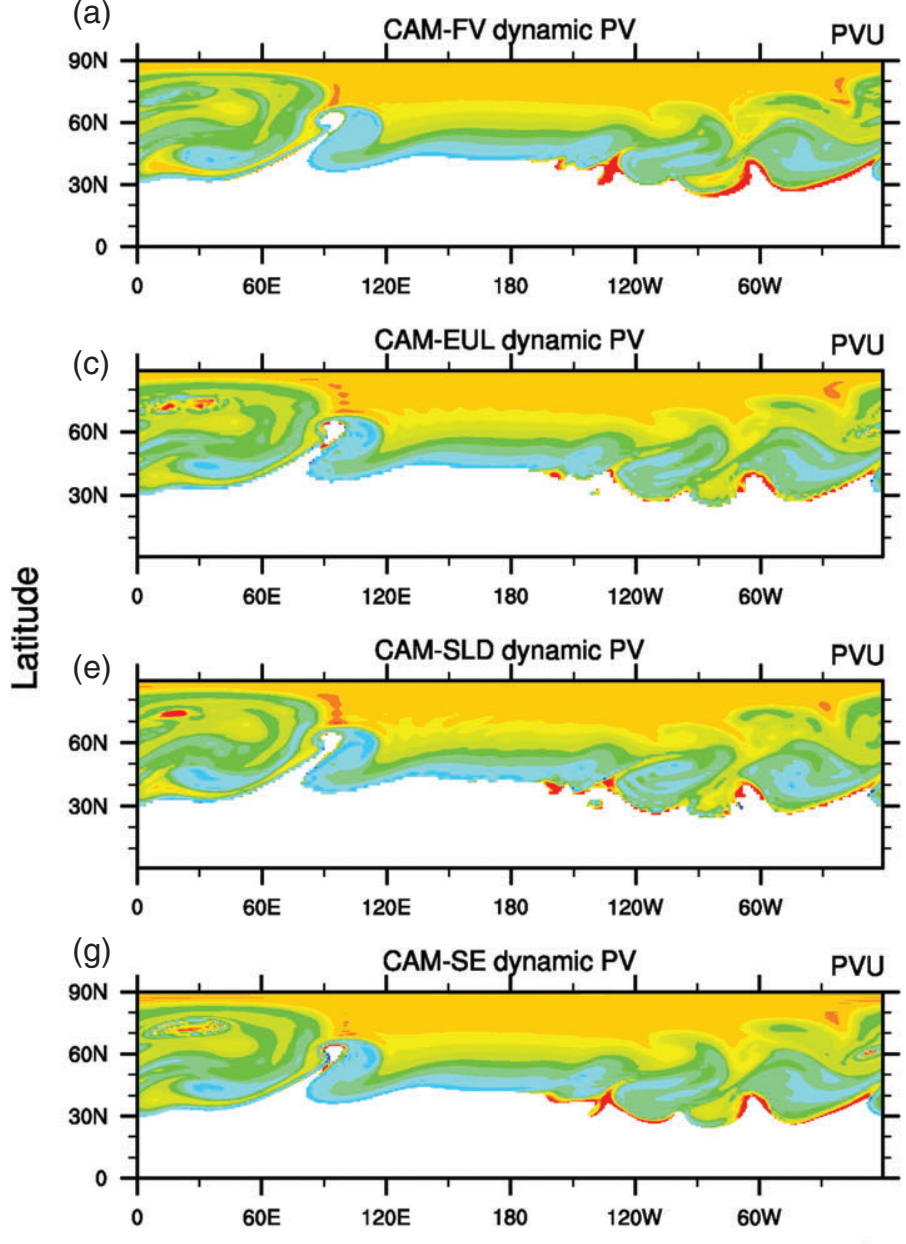

(b)
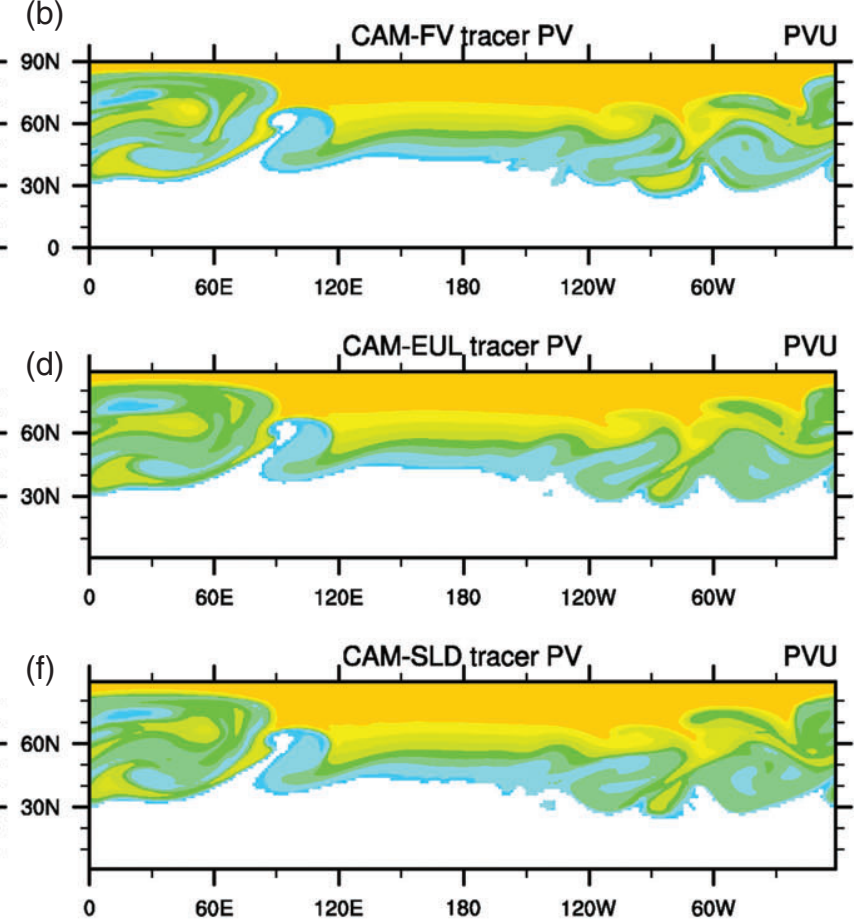

(h)

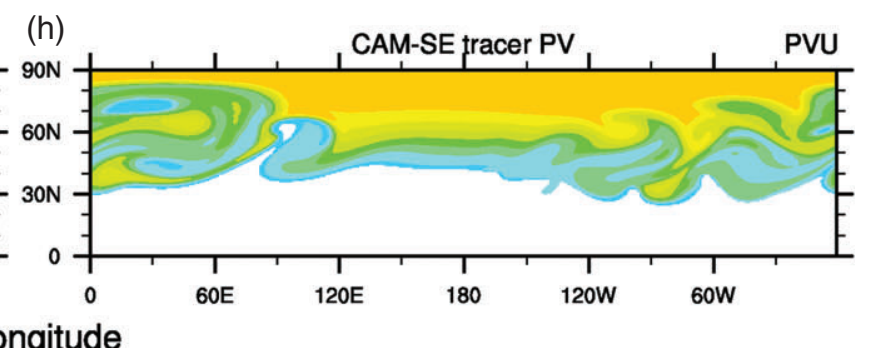

Longitude

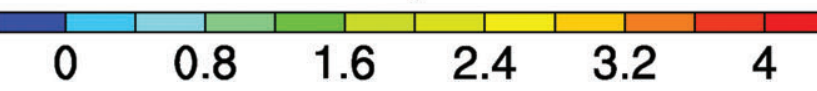

Figure 10. A snapshot at day 15 of the (a, c, e, g) dynamic and (b, d, f, h) tracer PV (PVU) at the $285 \mathrm{~K}$ isentropic level for the four dynamical cores to illustrate the differences in their treatment of the dynamics and tracers. The models are run with the configuration described in Table 1 . The white space is where the $285 \mathrm{~K}$ isentropic level has intersected the Earth's surface.

$160^{\circ} \mathrm{W}$ and $40^{\circ} \mathrm{N}$ ) are present in all four dynamical cores. They grow in magnitude with increased resolution (not shown). In the simulations reported here, the value of this spurious PV is nearly one order of magnitude larger than the maximum of the tracer PV at the same time stamp and isentropic level. Such PV anomalies are particularly worrisome in the region near $20^{\circ} \mathrm{E}$ and $75^{\circ} \mathrm{N}$ where the local minimum of the tracer PV for all the dynamical cores has become a local maximum for the dynamic PV, with the exception of CAM-FV. In addition, significant negative PV appears in these regions indicating a static instability, although this is difficult to visualize as these features are too small. Such PV anomalies near a boundary have been observed previously in reduced models (Nakamura and Held, 1989; Garner et al., 1992; Schneider et al., 2002) and in a full general circulation model (Konor and Arakawa, 1997; Woolings, 2004). In each case, a different driving mechanism for these anomalies is proposed, but there is no consensus as to their source. Using the evaluation technique developed here, we intend to pursue this phenomenon in greater detail.

After the generation of large values of dynamical PV, even prior to the intersection of the isentropic surfaces and the ground, the consistency between the dynamic PV and the tracer PV breaks down (Figure 8). This suggests either a numerical artifact or physical phenomena which are not adequately represented in our discrete model. The tracer PV develops neither the large values nor the small-scale structure of the dynamic PV. We draw an analogue to the atmosphere where pdfs and the correlative behaviour of PV and tracers have been used to isolate and quantify mixing (Rood et al., 1997, 2000). A challenge and a limit of using PV in these applications is that noise is amplified by the derivatives in the PV calculation. The results here suggest that there are similar limits even in the calculation of dynamical PV in a relatively simple nonlinear flow. The results also suggest that in climate models carrying a PV-like tracer for diagnostic applications is likely to offer an advantage in determining mixing and the identification of dynamical features.

This point is generalized by considering the importance of the effect of small-scale nonlinear flows on the transport of chemical trace species, specific humidity and cloud liquid water. For example, Ovtchinnikov and Easter (2009) have argued (with respect to cloud-aerosol interactions) that it is important to verify the consistency between the evolution of the winds and the integration of tracers via those winds. Otherwise, the unresolved and underresolved subgrid-scale effects, which are mimicked by the diffusive processes in the dynamical core, will not be correctly represented in the tracer transport scheme. Such inconsistencies have detrimental effects on the overall simulation quality, and affect not just the passive tracers but also cloud schemes and other physical parametrizations through their effect on chemical constituents.

\section{Appendix}

\section{The Initial Tracer PV}

The expressions for the velocity components, temperature and relative vorticity for the baroclinic wave test case are given in Jablonowski and Williamson (2006a) (their Eqs (2)-(6) 
and (12)). Here we only review the new components necessary to analytically compute the initial tracer PV field. The definition of the hydrostatic variant of Ertel's potential vorticity (Hoskins et al., 1985) on levels of constant pressure is

$$
q=g\left\{\frac{1}{a \cos \phi} \frac{\partial v}{\partial p}\left(\frac{\partial \theta}{\partial \lambda}\right)_{p}-\frac{1}{a} \frac{\partial u}{\partial p}\left(\frac{\partial \theta}{\partial \phi}\right)_{p}+\left(f+\zeta_{p}\right)\left(-\frac{\partial \theta}{\partial p}\right)\right\},
$$

where $a$ is the Earth's radius, $\zeta_{p}$ the relative vorticity, and $\lambda$ longitude. The subscript $p$ denotes a constant pressure level. We initialize the tracer with the absolute value of $\mathrm{PV}(q=|P V|)$ to avoid negative initial PV tracer values in the Southern Hemisphere. Note that the initial potential temperature does not vary in $\lambda, v$ is zero and that $\partial p$ can be expressed as $p_{0} \partial \eta$. The underlying relationship $p=\eta p_{0}$ is only valid under the special condition that the initial surface pressure $p_{s}$ is equal to the constant reference pressure $p_{0}=1000 \mathrm{hPa}$ which is the case here. The symbol $\eta$ denotes the orography-following pressurebased hybrid coordinate (Simmons and Burridge, 1981). Using all simplifications in Eq. (A1), the initial absolute value of the PV tracer yields

$$
\begin{aligned}
q(\lambda, \phi, \eta) & =|\operatorname{PV}(\lambda, \phi, \eta)| \\
& =\left|\frac{g}{p_{0}}\left\{-\frac{1}{a} \frac{\partial u}{\partial \eta}\left(\frac{\partial \theta}{\partial \phi}\right)_{p}-\left(f+\zeta_{p}\right) \frac{\partial \theta}{\partial \eta}\right\}\right| .
\end{aligned}
$$

To ease the reproducibility of the PV assessments we provide the analytical expressions for the derivative terms in Eq. (A2). All symbols and physical constants used below are defined in Jablonowski and Williamson (2006a) and are not all repeated here. The terms are

$$
\begin{aligned}
\frac{\partial u}{\partial \eta}= & -u_{0} \sin ^{2}(2 \phi) \frac{3 \pi}{4} \cos ^{1 / 2} \eta_{\mathrm{v}} \sin \eta_{\mathrm{v}} \\
\frac{\partial \theta}{\partial \eta}= & \frac{\partial \bar{\theta}}{\partial \eta}+\frac{3}{4} \frac{\pi u_{0}}{R_{d}}\left(1-\frac{R_{d}}{c_{p}}\right) \eta^{-R_{d} / c_{p}} \sin \eta_{\mathrm{v}} \cos ^{1 / 2} \eta_{\mathrm{v}} Y \\
& +\frac{3}{8} \frac{\pi^{2} u_{0}}{R_{d}} \eta^{1-R_{d} / c_{p}} \cos ^{3 / 2} \eta_{\mathrm{v}} Y \\
& -\frac{3}{16} \frac{\pi^{2} u_{0}}{R_{d}} \eta^{1-R_{d} / c_{p}} \sin ^{2} \eta_{\mathrm{v}} \cos ^{-1 / 2} \eta_{\mathrm{v}} Y \\
& -\frac{9}{8} \frac{\pi^{2} u_{0}^{2}}{R_{d}} \eta^{1-R_{d} / c_{p}} \sin ^{2} \eta_{\mathrm{v}} \cos \eta_{\mathrm{v}} \\
& \times\left\{-2 \sin ^{6} \phi\left(\cos ^{2} \phi+\frac{1}{3}\right)+\frac{10}{63}\right\}
\end{aligned}
$$

with $\eta_{\mathrm{v}}=0.5\left(\eta-\eta_{0}\right) \pi, \eta_{0}=0.252$, and

$$
\begin{aligned}
Y= & \left\{-2 \sin ^{6} \phi\left(\cos ^{2} \phi+\frac{1}{3}\right)+\frac{10}{63}\right\} 2 u_{0} \cos ^{3 / 2} \eta_{\mathrm{v}} \\
& +\left\{\frac{8}{5} \cos ^{3} \phi\left(\sin ^{2} \phi+\frac{2}{3}\right)-\frac{\pi}{4}\right\} a \Omega .
\end{aligned}
$$

In addition, the vertical gradient of the potential temperature, $\partial \bar{\theta} / \partial \eta$, is given by

$$
\left\{\begin{array}{cc}
T_{0} R_{d}\left(\frac{\Gamma}{g}-\frac{1}{c_{p}}\right) \eta^{R_{d}\left(\Gamma / g-1 / c_{p}\right)-1} & \text { for } \eta_{s}=1 \geq \eta \geq \eta_{t} \\
T_{0} R_{d}\left(\frac{\Gamma}{g}-\frac{1}{c_{p}}\right) \eta^{R_{d}\left(\Gamma / g-1 / c_{p}\right)-1} & \\
-\Delta T\left\{5\left(\eta_{t}-\eta\right)^{4} \eta^{-R_{d} / c_{p}}\right. & \\
\left.+\frac{R_{d}}{c_{p}}\left(\eta_{t}-\eta\right)^{5} \eta^{-R_{d} / c_{p}-1}\right\} & \text { for } \eta_{t}>\eta .
\end{array}\right.
$$

The derivative of the potential temperature with respect to the latitudinal direction on a constant pressure level is given by

$$
\begin{aligned}
\left(\frac{\partial \theta}{\partial \phi}\right)_{p}= & \frac{3}{4} \frac{\pi u_{0}}{R_{d}} \eta^{1-R_{d} / c_{p}} \sin \eta_{\mathrm{v}} \cos ^{1 / 2} \eta_{\mathrm{v}} \\
& \times\left[2 u _ { 0 } \operatorname { c o s } ^ { 3 / 2 } \eta _ { \mathrm { v } } \left\{-12 \cos \phi \sin ^{5} \phi\left(\cos ^{2} \phi+\frac{1}{3}\right)\right.\right. \\
\left.+4 \cos \phi \sin ^{7} \phi\right\} & \\
+a \Omega & \left\{-\frac{24}{5} \sin \phi \cos ^{2} \phi\left(\sin ^{2} \phi+\frac{2}{3}\right)\right. \\
& \left.\left.+\frac{16}{5} \cos ^{4} \phi \sin \phi\right\}\right]
\end{aligned}
$$

The appendix of Jablonowski and Williamson (2006a) outlines how the initial conditions can be computed for models with height-based or isentropic-based vertical coordinates, if needed. This analytic tracer PV initialization is also valid for nonhydrostatic dynamical cores that utilize the shallow-atmosphere approximation.

\section{Acknowledgements}

We would like to thank two anonymous reviewers and the editors who handled this article, whose comments greatly enhanced the presentation, as well as P H Lauritzen, C-Y Hsu, C M Zarzycki, K A Reed, P A Ullrich and M A Taylor for some helpful discussions and computational assistance. We acknowledge high-performance computing support from the Center for Advanced Computing at the University of Michigan and Yellowstone provided by NCAR's Computational and Information Systems Laboratory, sponsored by the National Science Foundation. This work was supported in part by the US Department of Energy's SciDAC program under grants DE-FG02-07ER64446 and DE-SC0006684, and through the LANL/LDRD Program as well as the National Science Foundation Award DGE-0221611.

\section{References}

Babiano A, Provenzale A. 2007. Coherent vortices and tracer cascades in two-dimensional turbulence. J. Fluid Mech. 574: 429-448.

Brennan MJ, Lackmann GM, Mahoney KM. 2007. Potential vorticity (PV) thinking in operations: The utility of non-conservation. Weather and Forecasting 23: 168-182.

Chagnon JM, Gray SL. 2009. Horizontal potential vorticity dipoles on the convective storm scale. Q. J. R. Meteorol. Soc. 135: 1392-1408.

Chagnon JM, Gray SL, Methven J. 2013. Diabatic processes modifying potential vorticity in a North Atlantic cyclone. Q. J. R. Meteorol. Soc. 139: 1270-1282.

Charney JG, Stern ME. 1962. On the stability of internal baroclinic jets in a rotating atmosphere. J. Atmos. Sci. 19: 159-172.

Colella P, Woodward PR. 1984. The Piecewise Parabolic Method (PPM) for gas-dynamical simulations. J. Comput. Phys. 54: 174-201.

Davis CA, Stoelinga MT, Kuo YH. 1993. The integrated effect of condensation in numerical simulations of extra-tropical cyclogenesis. Mon. Weather Rev. 121: 2309-2330.

Dennis JM, Fournier A, Spotz WF, St-Cyr A, Taylor MA, Thomas SJ, Tufo H. 2005. High-resolution mesh convergence properties and parallel efficiency of a spectral element atmospheric dynamical core. Int. J. High Perform. Comput. Appl. 19: 225-235.

Dennis JM, Edwards J, Evans KJ, Guba O, Lauritzen PH, Mirin AA, StCyr A, Taylor MA, Worley PH. 2012. CAM-SE: A scalable spectral element dynamical core for the Community Atmosphere Model. Int. J. High Perform. Comput. Appl. 26: 74-89.

Ertel H. 1942. Ein neuer hydrodynamischer Wirbelsatz. Meteorol. Z. 59: $271-281$.

Garner ST, Nakamura N, Held IM. 1992. Nonlinear equilibrium of twodimensional Eady waves: A new perspective. J. Atmos. Sci. 49: 1984-1996.

Gibbon JD, Holm DD. 2010. 'Stretching and folding diagnostics in solutions of the three-dimensional Euler and Navier-Stokes equations'. arXiv: 1012.359v1.

Gray SL. 2006. Mechanisms of midlatitude cross-tropopause transport using a potential vorticity budget approach. J. Geophys. Res. 111: D17113, doi: 10.1029/2005JD006259. 
Haynes PH, McIntyre ME. 1987. On the evolution of vorticity and potentia vorticity in the presence of diabatic heating and frictional or other forces. $J$ Atmos. Sci. 44: 828-841.

Haynes PH, McIntyre ME. 1990. On the conservation and impermeability theorems for potential vorticity. Mon. Weather Rev. 47: 2021-2031.

Hoskins BJ, McIntyre ME, Robertson AW. 1985. On the use and significance of isentropic potential vorticity maps. Q. J. R. Meteorol. Soc. 111: 877-946.

Jablonowski C, Williamson DL. 2006a. A baroclinic instability test case for atmospheric model dynamical cores. Q. J. R. Meteorol. Soc. 132: 2943-2975.

Jablonowski C, Williamson DL. 2006b. 'A baroclinic wave test case for dynamical cores of general circulation models: Model intercomparisons'. Technical Note NCAR/TN-469+STR. National Center for Atmospheric Research: Boulder, CO.

Jablonowski C, Williamson DL. 2011. The pros and cons of diffusion, filters, and fixers in atmospheric general circulation models. In Numerical Techniques for Global Atmospheric Models, Lauritzen PH, Jablonowski C, Taylor MA Nair RD. (eds.) Lecture Notes in Science and Engineering 80: 381-493. Springer: Berlin.

Joeckel P, von Kuhlmann R, Lawrence MG, Steil B, Brenninkmeijer CAM, Crutzen PJ, Rasch PJ, Eaton B. 2001. On a fundamental problem in implementing flux-form advection schemes for tracer transport in threedimensional general circulation and chemistry transport models. Q. J. $R$ Meteorol. Soc. 127: 1035-1052.

Kent J, Jablonowski C, Whitehead JP, Rood RB. 2012a. Assessing trace transport algorithms and the impact of vertical resolution in a finite-volum dynamical core. Mon. Weather Rev. 140: 1620-1638.

Kent J, Jablonowski C, Whitehead JP, Rood RB. 2012b. Downscale cascades in tracer-transport test cases: An intercomparison of the dynamical cores in the Community Atmosphere Model CAM5. Geosci. Model Dev. 5: 1517-1530.

Kent J, Ullrich PA, Jablonowski C. 2014. Dynamical Core Mode Intercomparison Project: Tracer transport test cases. Q. J. R. Meteorol. Soc., doi: 10.1002/qj.2208.

Konor CS, Arakawa A. 1997. Design of an atmospheric model based on generalized vertical coordinate. Mon. Weather Rev. 125: 1649-1673.

Lamarque J-F, Kinnison DE, Hess PG, Vitt FM. 2008. Simulated lowe stratospheric trends between 1970 and 2005: Identifying the role of climate and composition changes. J. Geophys. Res. 113: D12301, doi: 10.1029/2007JD009277.

Lauritzen PH, Ullrich PA, Nair RD. 2011. Atmospheric transport schemes: Desirable properties and a semi-Lagrangian view on finite-volume discretizations. In Numerical Techniques for Global Atmospheric Models, Lauritzen PH, Jablonowski C, Taylor MA, Nair RD. (eds.) Lecture Notes in Science and Engineering 80: 187-248. Springer: Berlin.

Lee SM, Yoon SC, Byun DW. 2004. The effect of mass inconsistency of the meteorological field generated by a common meteorological model on air quality modeling. Atmos. Environ. 38: 2917-2926.

Lin SJ. 2004. A 'vertically Lagrangian' finite-volume dynamical core for globa models. Mon. Weather Rev. 132: 2293-2307.

Lin SJ, Rood RB. 1996. Multidimensional flux-form semi-Lagrangian transport scheme. Mon. Weather Rev. 124: 2046-2070.

Lin SJ, Rood RB. 1997. An explicit flux-form semi-Lagrangian shallow-water model on the sphere. Q. J. R. Meteorol. Soc. 123: 2477-2498.

Nair RD, Lauritzen PH. 2010. A class of deformational flow test-cases for linear transport problems on the sphere. J. Comput. Phys. 229: 8868-8887.

Nakamura N, Held IM. 1989. Nonlinear equilibration of two-dimensional Eady waves. J. Atmos. Sci. 46: 3055-3064

Neale RB, Chen CC, Gettelman A, Lauritzen PH, Park S, Williamson DL, Conley AJ, Garcia R, Kinnison D, Lamarque JF, Marsh D, Mills M, Smith AK, Tilmes S, Vitt F, Cameron-Smith P, Collins WD, Iacono MJ, Easte RC, Liu X, Ghan SJ, Rasch PJ, Taylor MA. 2010. 'Description of the NCAR Community Atmosphere Model (CAM 5.0)', Technical Note NCAR/TN486+STR. National Center for Atmospheric Research: Boulder, CO.

Newman PA, Schoeberl MR, Plumb RA, Rosenfield JE. 1988. Mixing rates calculated from potential vorticity. J. Geophys. Res. 93: 5221-5240, doi: 10.1029/JD093iD05p05221

Ohkitani K. 1991. Wave number space dynamics of enstrophy cascade in forced two-dimensional turbulence. Phys. Fluids A 3: 1598-1611.

Ovtchinnikov M, Easter RC. 2009. Nonlinear advection algorithms applied to interrelated tracer: Errors and implications for modeling aerosol-cloud interactions. Mon. Weather Rev. 137: 632-644.

Rasch PJ, Coleman DB, Mahowald N, Williamson DL, Lin SJ, Boville BA, Hes P. 2006. Characteristics of atmospheric transport using three numerical formulations for atmospheric dynamics in a single GCM framework. J. Clim. 19: 2243-2266.

Reed KA, Jablonowski C. 2012. Idealized tropical cyclone simulations of intermediate complexity: A test case for AGCMs. J. Adv. Model. Earth Syst. 4: M04 001

Rood RB. 2011. A perspective on the role of the dynamical core in the development of weather and climate models. In Numerical Techniques for Global Atmospheric Models, Lauritzen PH, Jablonowski C, Taylor MA, Nair RD. (eds.) Lecture Notes in Science and Engineering 80: 523-544. Springer: Berlin.

Rood RB, Douglass AR, Cerniglia MC, Read WG. 1997. Synoptic-scale mass exchange from the troposphere to the stratosphere. J. Geophys. Res. 102: 23467-23485, doi: 10.1029/97JD01598.

Rood RB, Douglass AR, Cerniglia MC, Sparling LC, Nielsen JE. 2000. Seasonal variability of middle-latitude ozone in the lowermost stratosphere derived from probability distribution functions. J. Geophys. Res. 105: 17793-17805, doi: 10.1029/2000JD900142.

Salmon R. 1998. Lectures on Geophysical Fluid Dynamics. Oxford University Press: Oxford, UK.

Schneider T, Held IM, Garner ST. 2002. Boundary effects in potential vorticity dynamics. J. Atmos. Sci. 60: 1024-1040.

Simmons AJ, Burridge DM. 1981. An energy and angular-momentum conserving vertical finite-difference scheme and hybrid vertical coordinate. Mon. Weather Rev. 109: 758-766.

Stoelinga MT. 1996. A potential vorticity-based study of the role of diabatic heating and friction in a numerically simulated baroclinic cyclone. Mon. Weather Rev. 124: 849-874.

Taylor MA. 2011. Conservation of mass and energy for the moist atmospheric primitive equations on unstructured grids. In Numerical Techniques for Global Atmospheric Models, Lauritzen PH, Jablonowski C, Taylor MA, Nair RD. (eds.) Lecture Notes in Science and Engineering 80: 357-380. Springer: Berlin.

Taylor MA, Fournier A. 2010. A compatible and conservative spectral element method on unstructured grids. J. Comput. Phys. 229: 5879-5895.

Taylor MA, Tribbia J, Iskandarani M. 1997. The spectral element method for the shallow-water equations on the sphere. J. Comput. Phys. 130: 92-108.

Taylor MA, St-Cyr A, Fournier A. 2009. A non-oscillatory advection operator for the compatible spectral element method. In Computational Science D ICCS 2009, Part II, Allen G. (ed.) Lecture Notes in Computer Science 5545: 273-282. Springer: Berlin.

van Leer B. 1974. Towards the ultimate conservative difference scheme, II Monotonicity and conservation combined in a second order scheme. $J$. Comput. Phys. 14: 361-370.

van Leer B. 1977. Towards the ultimate conservative difference scheme, III Upstream centered finite-difference schemes for ideal compressible flow. J. Comput. Phys. 23: 263-275.

Wan H, Wang B, Yu YQ, Yu RC. 2006. 'Development and validation of the grid point atmospheric model of IAP LASG (GAMIL)', Technical report 16. LASG, Institute of Atmospheric Physics, Chinese Academy of Sciences: Beijing.

Wang B, Wan H, Li ZZ, Zhang X, Yu RC, Yu YQ, Liu HT. 2004. Design of a new dynamical core for global atmospheric models based on some efficient numerical methods. Sci. China A 47 (Suppl.): 4-21.

Whitehead JP, Jablonowski C, Rood RB, Lauritzen PH. 2011. A stability analysis of divergence damping on a latitude-longitude grid. Mon. Weather Rev. 139: $2976-2993$.

Williamson DL. 2007. The evolution of dynamical cores for global atmospheric models J. Meteorol. Soc. Jpn. 85B: 241-269.

Williamson DL. 2008. Equivalent finite volume and Eulerian spectral transform horizontal resolutions established from aqua-planet simulations. Tellus $A$ 60: 839-847.

Woolings T. 2004. 'Entropy and potential vorticity in dynamical core atmosphere models', PhD thesis. University of Reading: Reading, UK.

Zapotocny TH, Lenzen AJ, Johnson DR, Reames FM, Politowicz PA, Schaak TK 1996. Joint distributions of potential vorticity and inert trace constituent in CCM2 and UW $\theta-\sigma$ model simulations. Geophys. Res. Lett. 23: 2525-2528, doi: 10.1029/96GL02298.

Zhang K, Wan H, Wang B, Zhang M. 2008. Consistency problem with tracer advection in the atmospheric model GAMIL. Adv. Atmos. Sci. 25: 306-318.

Ziv B, Alpert P. 1994. Isobaric to isentropic interpolation errors and implication to potential vorticity analysis. J. Appl. Meteorol. 33: 694-703. 